\title{
Synthesis of a spacer-containing repeating unit of the capsu- lar polysaccharide of Streptococcus pneumoniae type 23F
}

\author{
Augusta M. P. van Steijn, Johannis P. Kamerling, and Johannes F. G. Vliegenthart \\ Bijvoet Center, Department of Bio-Organic Chemistry, Utrecht University, P.O. Box 80.075, NL-3508 TB \\ Utrecht (The Netherlands)
}

(Received June 11th. 1990; accepted for publication, August 9th, 1990)

\section{ABSTRACT}

The synthesis is reported of 3-aminopropyl 4- $O$-(4-O- $\beta$-D-glucopyranosyl-2- $O$ - $\alpha$-L-rhamnopyranosyl- $\beta$-D-galactopyranosyl)- $\beta$-L-rhamnopyranoside 3'-(glycer-2-yl sodium phosphate) (25B), which represents the repeating unit of the capsular polysaccharide of Streptococcus pneumoniae type $23 \mathrm{~F}$ (American type 23$)(\{\rightarrow 4)-\beta$-D-Glcp-( $(1 \rightarrow 4)$-[Glycerol- $(2-\mathrm{P} \rightarrow 3)][x-\mathrm{L}-\mathrm{Rh}$ a $p-(1 \rightarrow 2)]-\beta$-D-Gal $p-(1 \rightarrow 4)-\beta$-L-Rhap $\left.-(1 \rightarrow\}_{n}\right)$. 2,4,6-Tri-O-acetyl-3-O-allyl- $\alpha$-D-galactopyranosyl trichloroacetimidate (5) was coupled with ethyl 2,3-di- $O$ benzyl-1-thio- $x$-L-rhamnopyranoside (6). Deacetylation of the resulting disaccharide derivative, followed by benzylidenation, and condensation with 2,3,4-tri- $O$-acetyl- $\alpha$-L-rhamnopyranosyl trichloroacetimidate (10) a fforded ethyl 4-O-[3-O-allyl-4,6-O-benzylidene-2-O-(2,3,4-tri- $O$-acetyl- $\alpha$-L-rhamnopyranosyl)- $\beta$-Dgalactopyranosyl]-2,3-di-O-benzyl-1-thio- $\alpha$-L-rhamnopyranoside (11). Deacetylation of 11 . followed by benzylation, selective benzylidene ring-opening, and coupling with 2,3,4,6-tetra- $O$-acetyl- $\alpha$-D-glucopyranosyl trichloroacetimidate (15) gave ethyl 4- $O$-[3-O-allyl-6- $O$-benzyl-4- $O-(2,3,4,6$-tetra- $O$-acetyl- $\beta$-D-glucopyranosyl)-2- $O$-(2,3,4-tri- $O$-benzyl- $\alpha$-L-Ihlainnopyranosyl) - $\beta$-D-galactopyranosyl]-2,3-di-O-benzyl-1-thio- $\alpha$ L-rhamnopyranoside (16). Deacetylation of $\mathbf{1 6}$ followed by benzylation, deallylation, and acetylation yielded ethyl 4- $O$-[3- $O$-acetyl-6- $O$-benzyl-4-O-(2,3,4,6-tetra- $O$-benzyl- $\beta$-D-glucopyranosyl)-2-O-(2,3,4-tri$O$-benzyl - $\alpha$-L-rhamnopyranosyl)- $\beta$-D -galactopyranosyl]-2,3-di - $O$-benzyl - I -thio - $\alpha$-L -rhamnopyranoside (20). The glycosyl bromide derived from $\mathbf{2 0}$, when coupled with 3-benzyloxycarbonylamino-1-propanol, gave the $\beta$-glycoside (21 $\beta$ ) as the major product. Deacetylation of $21 \beta$ followed by condensation with 1,3-di-O-benzylglycerol 2-(triethylammonium phosphonate) (27), oxidation, and deprotection, afforded $25 \beta$.

\section{INTRODUCTION}

Streptococcus pneumoniae can induce infections such as pneumonia, otitis media, and meningitis in human beings. A polysaccharide vaccine ${ }^{1}$ (Pneumovax 23 ) against pneumococcal diseases is available, which contains the capsular polysaccharides isolated from 23 species of $S$. pneumoniae. However, these polysaccharides are non-immunogenic in newborns and do not induce a long-lasting immunological memory (TIresponse), and the induction of tolerance is a scvere problem ${ }^{2}$. In the context of developing new vaccines, oligosaccharide conjugates (neoglycoproteins/neoglycolipids), produced from oligosaccharides obtained by synthesis or degradation of polysaccharides, are of interest. Oligosaccharides related to pneumococcal polysaccharides of types 3 (ref. 3), 6A/6B (refs. 4-6), 9V (ref. 7), 14 (refs. 8, 9), 19A (ref. 10), and 19F (ref. 
11) have been synthesised and others related to types 3, 6B. and 14 (refs. 12, 13, and 14 . respectively) have been obtained by degradation of the polysaccharides. We now report on the synthesis of an oligosaccharide fragment of the capsular polysalccharide of $S$ phemonia type 23F (American type 23). Three different structures 1.A IC have been published ${ }^{15}$ for this capsular polysaccharide. At the start of our project. structure $1 \mathrm{~A}$ (ref. 15 ) and its revised form $\mathbf{1 B}$ (ref. 16) were avalable. and phosphate-containing 1 riand tetra-saccharide fragments related to structure $\mathbf{1 B}$ were synthesised" When this work was completed. the revised structure $\mathbf{1 C}$ was published. The synthesis of nonphosphorylated di-and tri-saceharides related to $\mathbf{A}$ and $\mathbf{I B}$ has been deseribed". In order to obtain additional evidence for the structure $1 \mathrm{C} . \mathrm{H}^{3 / \mathrm{P}}$;elayed spin-echo difference spectroscopy (RESED) F $^{21}$ was applied in order to identify the sugar residue atached to the (glycerophosphate group. The resulting sub-spectrum (Fig. I) of the phosphorylated sugar residues in the capsular polysaccharide type 235 proves that the phosphodiester bridge is between glycerol and galactose. in agreement with the revised structure $1 \mathrm{C}$.

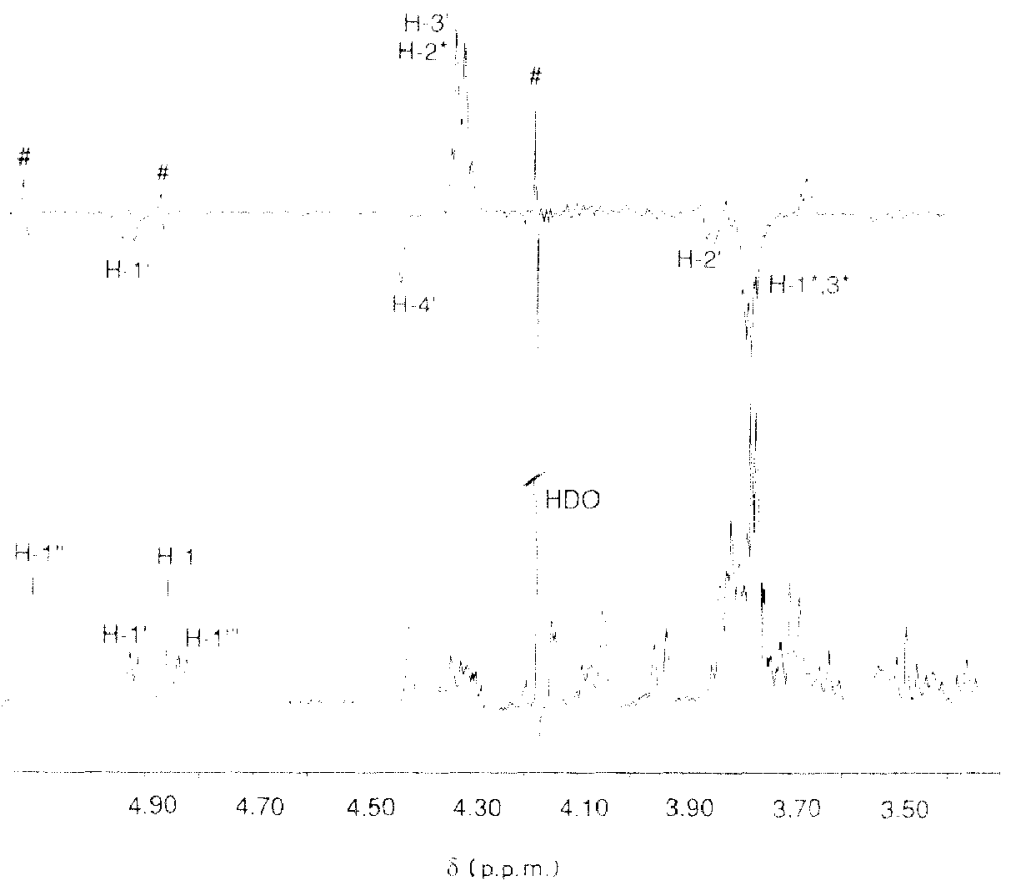

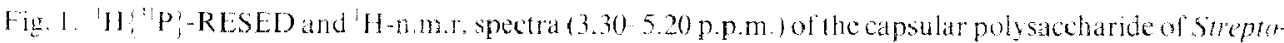

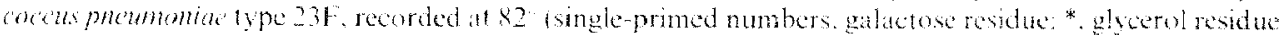
\#. stebtraction artefacts 


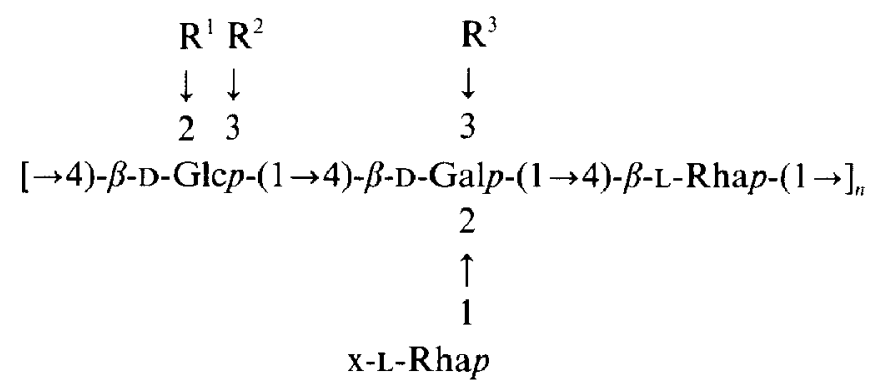

1A, $\mathbf{R}^{1}=$ glycerol 1-phosphate, $\mathrm{R}^{2}=\mathrm{R}^{3}=\mathrm{OH}, \mathrm{x}=\beta$

1B, $\mathrm{R}^{2}=$ phosphate, $\mathrm{R}^{1}=\mathrm{R}^{3}=\mathrm{OH}, \mathrm{x}=\alpha$

1C, $\mathrm{R}^{1}=\mathrm{R}^{2}=\mathrm{OH}, \mathrm{R}^{3}=$ glycerol 2-phosphate, $\mathrm{x}=\alpha$

We now report the synthesis of 3-aminopropyl 4-O-(4-O- $\beta$-D-glucopyranosyl-2$O$ - $\alpha$-L-rhamnopyranosyl- $\beta$-D-galactopyranosyl)- $\beta$-L-rhamnopyranoside 3 -(glycer-2-yl sodium phosphate) $(\mathbf{2 5 \beta})^{*}$, the spacer-coupled repeating unit of polysaccharide 1C.

\section{RESULTS AND DISCUSSION}

In the synthesis of $\mathbf{2 5 \beta}$, the selectively protected tetrasaccharide thioglycoside $\mathbf{2 0}$ was the key intermediate. Thioglycosides allow coupling to different aglycons, and oligosaccharide thioglycosides can be used in the stepwise synthesis of higher oligomers. Thioglycosides ${ }^{21}$ can be activated by thiophilic reagents or converted into the corresponding glycosyl bromides. Problems that can arise from the use of thioglycosides as acceptors have been reported, e.g., formation of the thioglycoside of the donor during coupling ${ }^{22}$. However, the use of glycosyl trichloroacetimidates as donors with thioglycosides as acceptors has given promising results ${ }^{18}$.

2,4,6-Tri- $O$-acetyl-3- $O$-allyl- $\alpha$-D-galactopyranosyl trichloroacetimidate (5) was used in the synthesis of 20 and 25B. Deacetylation of 7, followed by 4,6-O-benzylidenation allows a protected rhamnose residue to be attached at $\mathrm{C}-2$ and subsequent selective reductive ring-opening exposes $\mathrm{HO}-4$, to which a suitably protected glucose residue can be coupled $(\rightarrow \rightarrow \mathbf{1 8})$. The 3-O-allyl group can be removed selectively from 18, so that in a final stage the glycerol 2-phosphate unit can be introduced. For the synthesis of 5 , methyl $3-O$-allyl- $\beta$-D-galactopyranoside ${ }^{23}$ was acetylated $(\rightarrow 2,99 \%)$ and, after conversion of MeO-1 into AcO-1 by acetolysis ( $2 \%$ sulfuric acid in acetic anhydride for $1.5 \mathrm{~h}$ at $\left.0^{\circ}\right), \mathbf{3}$ was deacetylated at $\mathrm{C}-1$ using hydrazine acetate ${ }^{24}(\rightarrow \mathbf{4}, 28 \%$

\footnotetext{
* 3-Aminopropyl $4-O$-(4-O- $\beta$-D-glucopyranosyl-2- $O$ - $\alpha$-L-rhamnopyranosyl- $\beta$-D-galactopyranosyl)- $\beta$-L-

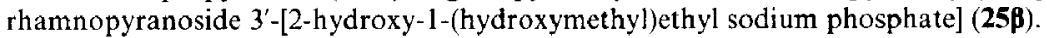


from 2 ) and the product was treated with trichloroacetonitrile in the presence of 1,8-diazabicyclo[5.4.0]undec-7-ene ${ }^{25}$ to afford 5 (78\%). Demethylation of 2 was more difficult than for methyl 2,4,6-tri-O-acetyl-3 $O$-benzyl- $\beta$-1-galactopyranoside ${ }^{1 k}$. Meth. ods applied in order to expose $\mathrm{HO}-1$, such as treatment with hydrochloric acid in acetic acid $^{26}$ or boron trichloride in dichloromethane ${ }^{27}$. yielded complex mixtures.

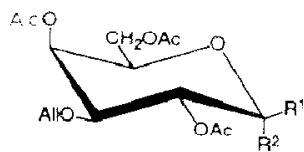

$2 P^{\prime}=O M E, R^{2}=H$

$3 A^{\prime} \cdot R^{2}: O A C_{1} H$

$4 R^{1} \cdot R^{2}-O H \cdot H$

$5 \mathrm{Fi}^{1} \because \mathrm{H}, \mathrm{R}^{2}: \mathrm{OCNHCOH}_{3}$

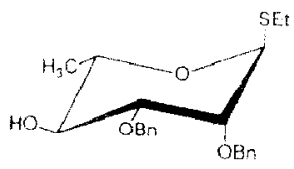

6

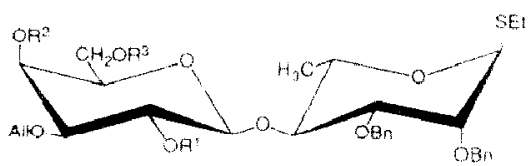

$7 B^{1} R^{2} \cdot A^{3} \cdot A C$

B) $A \cdot R^{2} \cdot R^{3} \cdot H$

o R $H, R^{2}, R^{3}$ CHPH

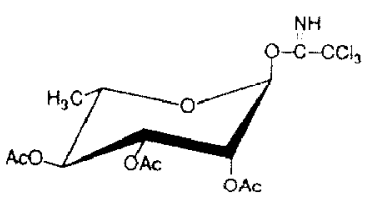

10

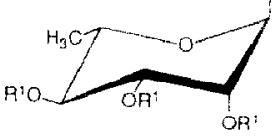

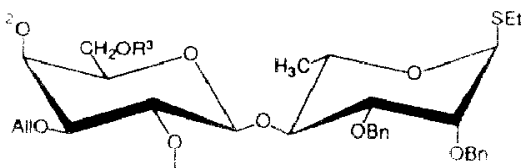

P 7

$$
\begin{aligned}
& 11 R^{1}=A c, R^{2}, A^{3}=C H P h \\
& 12 R^{1} \cdot H \cdot R^{2} R^{3}=C H P h \\
& 13 R^{4}=B \cap R^{2} \cdot R^{3}=C H P h \\
& 19 R^{1}=R^{3}-B \cap, R^{2} H
\end{aligned}
$$

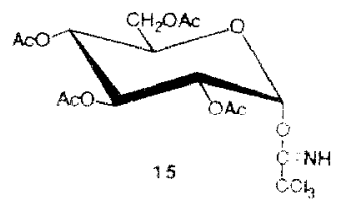

Condensation of 5 with ethyl 2,3-di-O-benzyl-1-thio- $x$-L-rhamnopyranoside " (6) in dichloromethane at -30 . using trimethylsilyl triflate as a catalyst. gave the disaccharide derivative 7 , which was easily separated from the starting compounds after deacetylation $(\rightarrow \mathbf{8}, 70 \%$ from $\mathbf{6})$. Compound $\mathbf{8}$ was benzylidenated $(\rightarrow \mathbf{9}, 79 \%)$. con densed with 2,3,4-tri-O-acetyl- $\gamma$-L-rhamnopyranosyl trichloroacerimidate $(10: \rightarrow 11)$ under the above conditions and the product was deacetylated to afford $12(78 \%$ from 9$)$. Sodium methoxide was removed by washing with water, because neutralisation with Dowex-50 $\left(\mathrm{H}^{+}\right)$resin causes debenzylidenation ${ }^{18}$. Benzylation of $12\left(\rightarrow \mathbf{1 3}, 93^{\circ} \%\right)$ fol lowed by selective opening of the 4,6-O-benzylidene ring with borane trimethylamine complex and aluminium(III) chloride in tetrahydrofuran yielded $14(69 \%)$.

The tetrasaccharide derivative 16 was prepared by coupling 2,3.4.6-tetra-Oacetyl- $x$-D-glucopyranosyl trichloroacetimidate ${ }^{29}(15)$ with $\mathrm{HO}-4$ of 14 . using rimethysilyl triflate as a catalyst. The formation of 16 occurred within a few minuts at - 10 :at $-30^{\circ}$, only one faster-moving compound was observed in t.l.c. which was converted into 16 when the temperature was raised to -10 . Deacetylation of 16 viclded $17\left(67^{\circ} \mathrm{n}\right.$ from 14). 

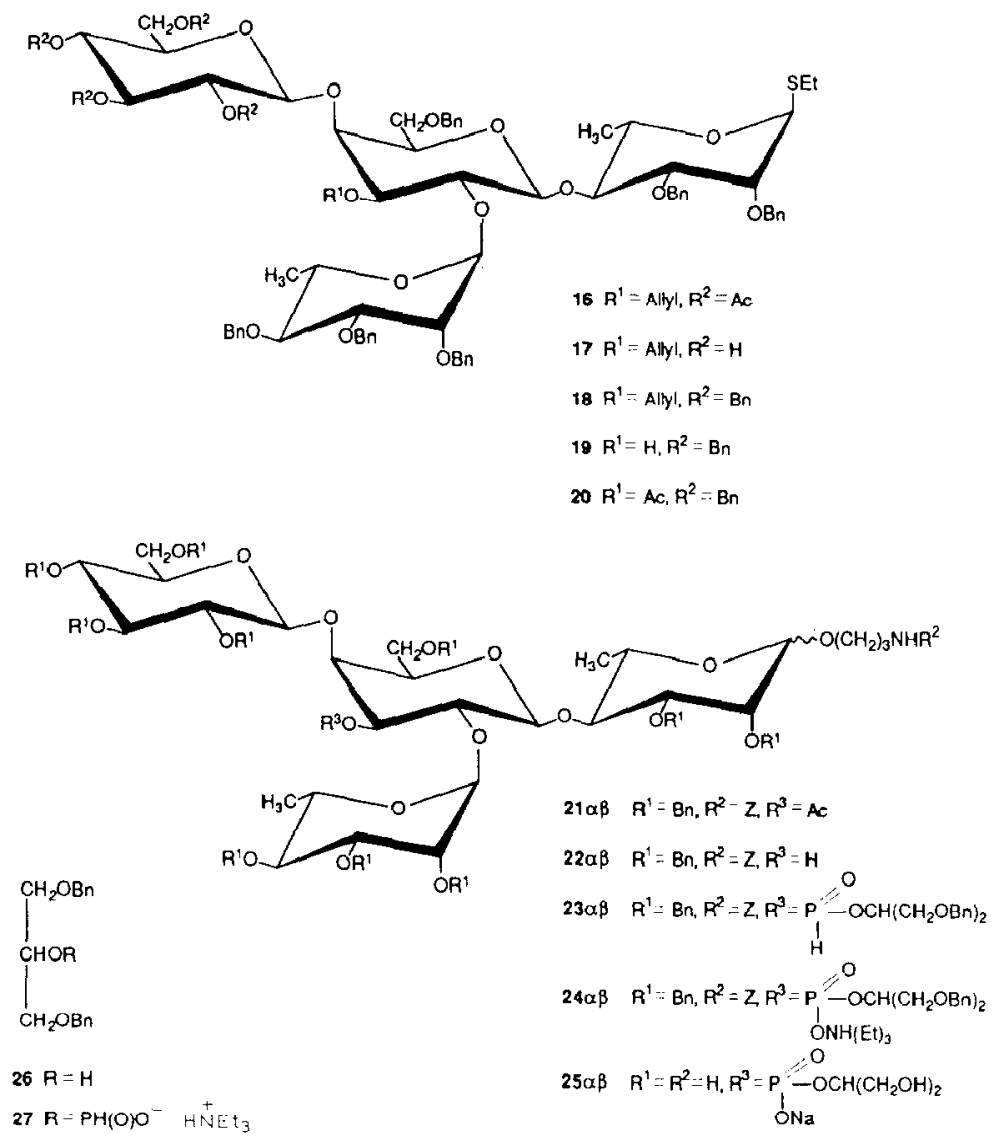

The tetrasaccharide derivative 17 was benzylated $(\rightarrow \mathbf{1 8}, 68 \%)$. The allyl group was removed from 18 using the Wilkinson catalyst ${ }^{30}$ in the presence of 1,4-diazabicyclo[2.2.2] octane followed by hydrolysis $(\rightarrow \mathbf{1 9}, 73 \%)$, and acetylation then gave $\mathbf{2 0}$ $(93 \%)$. The replacement of the 3-O-allyl group of the galactose moiety by an acetyl group $(\mathbf{1 8} \rightarrow \mathbf{2 0})$, before coupling to the intended spacer, was necessary because of the instability of the allyl group under the conditions applied for the transformation of the ethyl thioglycoside into a glycosyl bromide ${ }^{18}$ using bromine (see below). Deallylation in the absence of base afforded only $40 \%$ of 19 . The use of potassium tert-butoxide $^{31}$ to isomerise the allyl group gave complex mixtures (t.l.c.), as occurred when palladium chloride ${ }^{25.32}$ was used to remove the allyl group.

The key intermediate $\mathbf{2 0}$ was coupled to the spacer 3-benzyloxycarbonylamino-1propanol $^{33,34}$. Test reactions on mono- and di-saccharide thioglycosides showed that direct coupling mediated by methyl triflate yielded a considerable proportion of the $\alpha$-glycoside in addition to the desired $\beta$ form, but reaction with the corresponding glycosyl bromides, using the insoluble silver catalyst method ${ }^{35}$, seemed more promising. The coupling of the bromide of $\mathbf{2 0}$, generated in situ ${ }^{36}$ with copper(II) bromide- 
tetrabutylammonium bromide, to the spacer in dichloromethane, using silver silicate ${ }^{37}$ as a promoter, proceeded slowly and, after several days, only a small amount of product was formed. When the ethyl thioglycoside $\mathbf{2 0}$ was converted in to the glycosyl bromide using bromine ${ }^{38}$, subsequent condensation with 3-benzyloxycarbonylamino-1-propanol $^{13}$ in dichloromethane-toluene gave better results and yielded $21(64 \%)$ with the

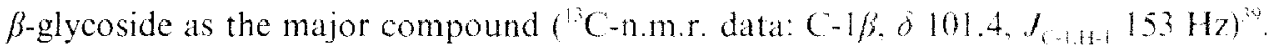
${ }^{13}$ C-N.m.r. spectroscopy indicated that only a small proportion of the $\alpha$-glycoside was formed. Subsequent deacetylation afforded $22 \alpha \beta(98 \%)$. with $\mathrm{HO}-3$ of the galactose residue available for formation of the phosphodiester bridge between the tetrasaccharide derivative and glycerol.

The phosphonate method ${ }^{4(-4)}$ chosen for the introduction of the phosphodiester bridge has the advantage that the intermediate phosphonic mono-and di-esters can be purified easily on silica gel. The phosphonic diester bridge was introduced by coupling the triethylammonium salt of the protected glycerol 2-phosphonate (27) with 22 . Compound 27 (89\% from 26) was prepared from 1,3-di-O-tritylglycerol ${ }^{\text {t4 }}$ by allylation. detritylation, benzylation, deallylation using potassium tert-butoxide $(\rightarrow 26)$, and reaction with 2-chloro-4H-1,3,2-benzodioxaphosphorin-4-one in pyridine ${ }^{41.42}$. Condensation of $\mathbf{2 7}$ with 22 in the presence of pivaloyl chloride in pyridine afforded the phosphonic diester $\mathbf{2 3} \alpha \boldsymbol{\beta}(82 \%)$. The 'H-n.m.r. spectrum of the product revealed equal amounts of two enantiomers of $23 \beta$ [ ${ }^{1} \mathrm{H}-\mathrm{n} . \mathrm{m} . \mathrm{r}$. data: $\delta 6.998\left(J_{p .3} 720 \mathrm{~Hz}\right)$ and $6.796\left(J_{\mathrm{P} . \mathrm{H}}\right.$ $727 \mathrm{IIz}$ ) $\mathrm{P}-\mathrm{H}]^{41}$. Mild oxidation of 23 with iodine in water pyridine $(\rightarrow \mathbf{2 4 \alpha \beta} .91 \%)$, followed by debenzyloxycarbonylation debenzylation and reatment with Dowex-50 $\left(\mathrm{Nat}^{+}\right)$resin, afforded $25 \alpha \boldsymbol{\beta}(57 \%)$ [ ${ }^{1} \mathrm{H}-\mathrm{n} . \mathrm{m} . \mathrm{r}$. data: $4.649(J,-0 \mathrm{~Hz}), \mathrm{H}-1$ of $\mathbf{2 5 \beta ;} \delta$ $5.045\left(J_{1,2} 1.2 \mathrm{~Hz}\right), \mathrm{H}-1$ of $\mathbf{2 5 \alpha} \alpha, \beta$ ratio $\left.1: 4\right)$. High performance anion-exchange chromatography, with pulsed amperometric detection (HPAE-PAD) on a CarboPac PAI column, was used to obtain pure $25 \beta$. The ${ }^{i} H-n, m, \pi$ data of $25 \beta$. obtained by $2 D$ $\mathrm{COSY}^{46}$ and $2 \mathrm{D} \mathrm{HOHAHA}$ measurements, are given in Table I. It may be noted that H-5 of the spacer-linked $\beta$-Rha residue resonated at 3.448 p.p.m., whereas $H-5$ of the terminal $\alpha$-Rha unit was observed at 4.096 p.p.m. in accord with literature data ${ }^{4}$. The RESED $^{20}$ spectrum of $25 \beta$ showed clearly that galactose and glycerol are involved in the

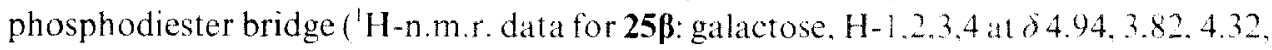
and 4.43, respectively; glycerol $\mathrm{H}-1 / 3,2$ at $\delta 3.77$ and 4.28. respectively). For comparison, the ${ }^{1} \mathrm{H}$-n.m.r. data of the bacterial polysaccharide have been included in Table I: there are several relatively small deviations that reflect the different microenvironments in the oligosaccharide and the polysaccharide. The reported ${ }^{\prime} \mathrm{C} \cdot \mathrm{n}, \mathrm{m} . \mathrm{r}$. data of the polysaccharide $[\delta 103.5,102.5,102.1$, and 101.7 (4 C-1),63.1 (CH,OH of glycerol). 62.5 and $62.1\left(\mathrm{CH}_{2} \mathrm{OH}\right.$ of galactose glucose $), 18.5$ and $17.9\left(\mathrm{CH}_{3}\right.$ of thamnose $\left.)\right]^{1 / 2}$ accord with those for $25 \beta$ ( $\delta 103.8,102.7,102.1,101.1,62.7,62.1,61,9,18.5$, and 17.8$)$.

Immunological studies of $25 \beta$ conjugated to protein will be reported elsewhere. 
TABLE I

$500-\mathrm{MHz}^{1} \mathrm{H}-\mathrm{N} . \mathrm{m}$. .r. chemical shift data ${ }^{a}$ for $25 \beta$ and for the polysaccharide ${ }^{17}\left(82^{\circ}\right)$ of $S$. pneumoniae type $23 \mathrm{~F}$.

\begin{tabular}{|c|c|c|c|c|c|}
\hline \multirow{3}{*}{ Residue } & \multirow{3}{*}{ Proton (J) } & \multicolumn{4}{|c|}{$\delta\left(\right.$ p.p.m., $\left(\mathrm{J}\right.$ in $\left.H_{z}\right)$} \\
\hline & & \multicolumn{2}{|l|}{$25 \beta$} & \multicolumn{2}{|c|}{ Polysaccharide } \\
\hline & & 4.648 & $(\sim 0)$ & 4.851 & $(1.1)$ \\
\hline & H-2 $\left(J_{2,3}\right)$ & 3.941 & $(3.0)$ & 4.043 & $(2.4)$ \\
\hline & $\mathrm{H}-3\left(J_{3,4}\right)$ & 3.80 & $(9.4)$ & 3.797 & $(9.5)$ \\
\hline & $\mathrm{II}-4\left(J_{4,5}\right)$ & 3.696 & $(9.4)$ & 3.688 & $(9.1)$ \\
\hline & $\mathrm{H}-5\left(J_{5,6}\right)$ & 3.448 & $(6.2)$ & 3.418 & $(5,8)$ \\
\hline & $\mathrm{H}-6$ & 1.359 & & 1.362 & \\
\hline \multirow[t]{8}{*}{$\beta-\mathrm{Gal}$} & $\mathrm{H}-1\left(J_{1,2}\right)$ & 4.938 & $(8.0)$ & 4.935 & $(7.6)$ \\
\hline & $\mathrm{H}-2\left(J_{23}\right)$ & 3.822 & $(9.6)$ & 3.824 & $(9.8)$ \\
\hline & $\mathrm{H}-3\left(J_{3,4}\right)$ & 4.325 & $(2.9)$ & 4.329 & $(2.8)$ \\
\hline & $\left(J_{\mathrm{H}, \mathrm{P}}\right)$ & & $(9.6)$ & & $(8.6)$ \\
\hline & $\mathrm{H}-4\left(J_{4,5}\right)$ & 4.427 & $(<1)$ & 4.416 & $(1.3)$ \\
\hline & H-5 $\left(J_{5,6 \mathrm{a}}\right)$ & n.d & & 3.803 & $(2.2)$ \\
\hline & $\mathrm{H}-6 \mathrm{a}\left(J_{6 \mathrm{a} .6 \mathrm{~b}}\right)$ & n.d. & & 3.787 & $(-11.5)$ \\
\hline & $\mathrm{H}-6 \mathrm{~b}\left(J_{5,6 \mathrm{~b}}\right)$ & n.d & & 3.749 & $(6.0)$ \\
\hline \multirow[t]{6}{*}{$x$-Rha } & $\mathrm{H}-1\left(J_{1.2}\right)$ & 5.075 & $(1.6)$ & 5.103 & $(1.0)$ \\
\hline & $\mathrm{H}-2\left(J_{2,3}\right)$ & 4.154 & $(3.5)$ & 4.141 & (3.8) \\
\hline & $\mathrm{H}-3\left(J_{3,4}\right)$ & 3.809 & $(9.8)$ & 3.814 & $(9.5)$ \\
\hline & $\mathrm{H}-4\left(J_{4,5}\right)$ & 3.470 & $(9.8)$ & 3.474 & $(9.5)$ \\
\hline & H-5 $\left(J_{5,6}\right)$ & 4.096 & $(6.3)$ & 4.072 & $(6.0)$ \\
\hline & $\mathrm{H}-6$ & 1.265 & & 1.267 & \\
\hline \multirow[t]{7}{*}{$\beta$-Glc } & H-1 $\left(J_{1,2}\right)$ & 4.813 & $(8.0)$ & 4.834 & $(7.4)$ \\
\hline & $\mathrm{H}-2\left(J_{2,3}\right)$ & 3.335 & $(9.4)$ & 3.357 & $(9.4)$ \\
\hline & $\mathrm{H}-3\left(J_{3,4}\right)$ & 3.540 & $(9.1)$ & 3.697 & $(9.1)$ \\
\hline & $\mathrm{H}-4\left(J_{4.5}\right)$ & 3.404 & $(9.8)$ & 3.618 & $(10.0)$ \\
\hline & $\mathrm{H}-5\left(J_{5,6 \mathrm{a}}\right)$ & 3.461 & $(2.2)$ & 3.519 & $(1.8)$ \\
\hline & H-6a $\left(J_{\text {fa } .6 \mathrm{~b}}\right)$ & 3.908 & $(-12.3)$ & 3.939 & $(-11.5)$ \\
\hline & $\mathrm{H}-6 \mathrm{~b}\left(J_{5,6 \mathrm{~b}}\right)$ & 3.746 & (n.d) & 3.796 & $(5.7)$ \\
\hline \multirow[t]{4}{*}{ Glycerol } & $\mathrm{H}-\mathrm{la}, \mathrm{lb}\left(J_{1,2}\right)$ & 3.77 & $(5.0)$ & 3.78 & $(3.9)$ \\
\hline & H-2 $\left(J_{1 \mathrm{a}, 1 \mathrm{~b}}=J_{3 \mathrm{a}, 3 \mathrm{~b}}\right)$ & 4.281 & (n.d.) & 4.290 & $(-12.2)$ \\
\hline & $\mathrm{H}-3 \mathrm{a}, 3 \mathrm{~b}\left(J_{2.3}\right)$ & 3.77 & $(5.0)$ & 3.74 & $(5.2)$ \\
\hline & $\left(J_{\mathrm{H}, \mathrm{P}}\right)$ & & $(8.0)$ & & $(9.8)$ \\
\hline \multirow[t]{4}{*}{ Spacer } & $\mathrm{H}-1$ & 3.122 & & & \\
\hline & $\mathrm{H}-2$ & 1.968 & & & \\
\hline & $\mathrm{H}-3 \mathrm{a}$ & 3.968 & & & \\
\hline & $\mathrm{H}-3 \mathrm{~b}$ & 3.791 & & & \\
\hline
\end{tabular}

"Chemical shifts are relative to the signal of sodium 4,4-dimethyl-4-silapentane-1-sulfonate (using internal acetone at $\delta 2.225$ p.p.m.) in $\mathrm{D}_{2} \mathrm{O}$.

EXPERIMENTAL

General methods. - 'H-N.m.r. spectra (360 and $500 \mathrm{MHz}$ ) were recorded at $25^{\circ}$ with a Bruker HX 360 or AM 500 spectrometer (Bijvoet Center, Utrecht University). 
2D double-quantum-filtered ' $\mathrm{H}{ }^{1} \mathrm{H}$ correlation spectra (2D DQF 'H H COSY) were recorded in the phase-sensitive mode ${ }^{36}$. and $2 \mathrm{D}$ homonuclear Harmann Hahn spectra (2D HOHAHA) with a MLEV-17 mixing sequence of $120 \mathrm{~ms}^{12}$. C-N.m.r. spectra (APT, $50 \mathrm{MHz}$ ) were recorded at 25 with a Bruker WP 200 spectromeler. Chemical shifts $(\delta)$ are given in p.p.m. relative to the signal for internal $\mathrm{Me}_{4} \mathrm{Si}(\mathrm{CDCl}$ ) or sodium 4,4-dimethyl-4-silapentane-1-sulfonate (D, O indirectly to internalacetone, 2.225 ) for 'H, and to the signal for intemal $\mathrm{Me}_{4} \mathrm{Si}\left(\mathrm{CDCl}_{3}\right)$ indirecty to CDC1, 876.9$)$ or axternal $\mathrm{Me}_{4} \mathrm{Si}(\mathrm{D}, \mathrm{O}$; indirectly to intemal acetone, 831.55$)$ for ${ }^{\circ} \mathrm{C}$

Column chromatography was performed on Kieselgel 60 (Merck. $<230$ mesh) and fractions were momtored by $1.1 . \mathrm{c}$. on K kesclgel of t... (Mcrk). Detection was effected by charring with sulfuric acid after examination under as. light. Optical rotations were measured at 20 with a Perkin Elmer 241 polarimeter asing a $10-\mathrm{cm}$ 1-mL cell. Melting points were determined with a Mettler F $5 /$ insttument. In the work-up procedures, washings were carried out three times with appropriate quantities of water or aqueous $5 \%$ sodium hydrogencarbonale unless indicated otherwise. Evaporations were conducted under reduced pressure at 40 ' thath. Nll solvents were divillod from appropriate drying agents.

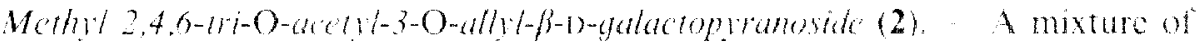

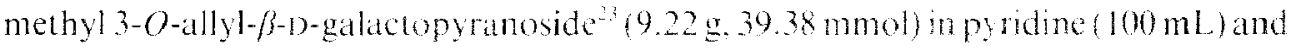
acetic anhydride $(100 \mathrm{~mL}$ was stirred for $16 \mathrm{~h}$ at room temperature, then co-concentrated with coluene $(3 \times 100 \mathrm{~mL})$, ethanol $(3 \times 100 \mathrm{~mL})$. and dichloromethane $3 \times 100$ $\mathrm{mL})$. Column chromatography $(85: 15$ dichloromethance ethyl acetate) of the residue

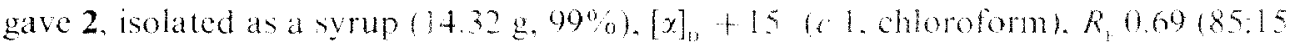

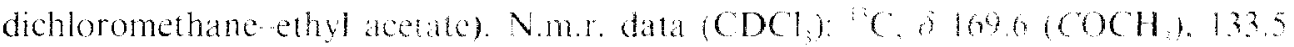
$\left(\mathrm{H}_{2} \mathrm{C}=(\mathrm{HCH} \mathrm{O}), 116.6 \mathrm{H} \mathrm{C}=\mathrm{CHCH} 0\right) .101 .5(\mathrm{C}-1), 76.2 .70 .3,69.9$. and 65.7 $(\mathrm{C}-2.3,4,5), 70,0(\mathrm{HC}=(\mathrm{CHCHO}, 61.3(\mathrm{C}-6) .56 .2(\mathrm{OCH}) .20 .4$ and $20.112 \mathrm{C})(3$

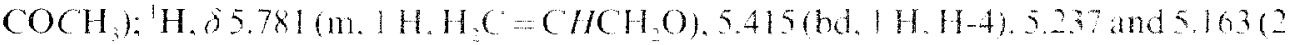
m. each $\left.\left.1 \mathrm{H}, H_{2} \mathrm{C}=\mathrm{CHCHO}, 5.085(\mathrm{dd}.) \mathrm{H}, \mathrm{H}-2\right) .4 .339(\mathrm{~d}.) \mathrm{H}, \mathrm{H}-1\right) .4 .169(\mathrm{~d} .2 \mathrm{H}$. H-6a,b). 4.124 and $3.904(2 \mathrm{~m}$, each $1 \mathrm{H}, \mathrm{H}, \mathrm{C}=\mathrm{CHCH}, \mathrm{O}) .3813(\mathrm{~b}, 1 \mathrm{H}, \mathrm{H}-5), 3.513$

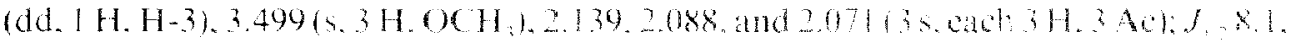
$J_{2,1}, 10.0, J_{3,4}, 5, J_{4,6}<1, J=6,9 \mathrm{H} \%$.

Anal. Cake for $\mathrm{C}_{1 \mathrm{i}} \mathrm{ll}, \mathrm{O}: \mathrm{C} .53 .33 ; \mathrm{H}, 6.71$. Found: $\mathrm{C} .53,22 ; \mathrm{H}, 6.89$

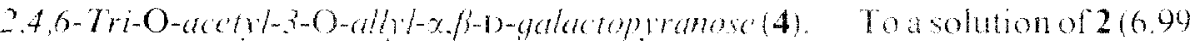
g. $19.42 \mathrm{mmol}$ in acetic anhydride $(73 \mathrm{~mL}$ ) at 0 was added a shotion of conc sulfuric acid $(2.9 \mathrm{~mL})$ in acetic anhydride $70 \mathrm{~mL}$. After $1.5 \mathrm{~h}$ at 0 . sodium aceate $12.1 \mathrm{~g} .147 .4$ mmol) was added. the mixume was concentrated. and a solution of the residue in ethyl acetate $(500 \mathrm{~mL})$ was washed. dried $\left(\mathrm{Na}_{2} \mathrm{SO}_{4}\right)$, filtered and concentrated wafford 3. which was used without further purification in the next step. To a solution of $3(3.01 \mathrm{~g})$ in dry N.N-dimethylformamide $(10 \mathrm{~mL})$ was added hydrazine acetale $1901 \mathrm{mg} .9 .77$ $\mathrm{mmol})$. After storage for 2 h at room temperature, the mixture was diluted with ethyl acelate $\left(300 \mathrm{~mL}\right.$ ), washed wh aqueous $5 \%$ sodium chloride dried ( Vas $80_{4}$ ) fillered. and concentrated. Column chromatogxaphy $(85.15$ dichoromethan ethy acetate) of the residue afforded 4 isolated as a syrup $\left(1.90 \mathrm{~g} .20^{\circ} \%\right), R \quad 0.4585 .15$ dichoro- 
methane ethyl acetate). ${ }^{13} \mathrm{C}-\mathrm{N} . m . r$. data $\left(\mathrm{CDCl}_{3}\right): \delta$ 170.4-170.2 $\left(\mathrm{COCH}_{3}\right), 134.0$ $\left(\mathrm{H}_{2} \mathrm{C}=\mathrm{CHCH}_{2} \mathrm{O}, \alpha\right), 133.7\left(\mathrm{H}_{2} \mathrm{C}=\mathrm{CHCH}_{2} \mathrm{O}, \beta\right), 117.2\left(\mathrm{H}_{2} \mathrm{C}=\mathrm{CHCH}_{2} \mathrm{O}, \beta\right), 117.0$ $\left(\mathrm{H}_{2} \mathrm{C}=\mathrm{CHCH}_{2} \mathrm{O}, \alpha\right), 95.6(\mathrm{C}-1 \beta), 90.3(\mathrm{C}-1 \alpha), 70.4\left(\mathrm{H}_{2} \mathrm{C}-\mathrm{CHCH}_{2} \mathrm{O}\right), 76.0,72.5,70.8$, and $66.0(\mathrm{C}-2,3,4,5, \beta), 72.0,70.0,67.3$, and $66.0(\mathrm{C}-2,3,4,5, \alpha), 62.1(\mathrm{C}-6 \alpha), 61.8(\mathrm{C}-6 \beta)$, $20.6=20.4\left(\mathrm{COCH}_{3}\right)$.

Anal. Calc. for $\mathrm{C}_{15} \mathrm{H}_{22} \mathrm{O}_{9}:$ C, 52.02; H, 6.40. Found: C, 51.71; $\mathrm{H}, 6.43$.

2,4,6-Tri-O-acetyl-3-O-allyl- $\alpha$-D-galactopyranosyl trichloroacetimidate (5). - To a solution of $4(1.90 \mathrm{~g}, 5.49 \mathrm{mmol})$ in dry dichloromethane $(70 \mathrm{~mL})$ and trichloroacetonitrile $(6.88 \mathrm{~mL}, 68.59 \mathrm{mmol})$ was added a solution of 1,8-diazabicyclo[5.4.0]undec-7ene $(0.82 \mathrm{~mL}, 5.49 \mathrm{mmol})$ in dichloromethane $(10 \mathrm{~mL})$ at $0^{\circ}$, and the mixture was stirred for $1 \mathrm{~h}$. The reaction was then complete (t.l.c., 9:1 dichloromethane = ethyl acetate, $R_{\mathrm{r}}$ $0.57)$ and the mixture was concentrated. Column chromatography $(9: 1$ dichloromethane $=$ ethyl acetate $)$ of the residue gave 5 , isolated as a yellow syrup $(2.09 \mathrm{~g}, 78 \%),[\alpha]_{\mathrm{D}}$ $+119^{\circ}$ (c 1, chloroform). N.m.r. data $\left(\mathrm{CDCl}_{3}\right):{ }^{13} \mathrm{C}, \delta 170.3-170.0\left(\mathrm{COCH}_{3}\right), 160.7$ $\left(\mathrm{OCNHCCl}_{3}\right), 133.9\left(\mathrm{H}_{2} \mathrm{C}=\mathrm{CHCH}_{2} \mathrm{O}\right), 117.2\left(\mathrm{H}_{2} \mathrm{C}=\mathrm{CHCH}_{2} \mathrm{O}\right), 93.9(\mathrm{C}-1), 90.9$ $\left(\mathrm{OCNHCCl}_{3}\right), 72.5,69.4 .68 .7$, and $66.7(\mathrm{C}-2,3,4,5), 70.6\left(\mathrm{H}_{2} \mathrm{C}=\mathrm{CHCH}_{2} \mathrm{O}\right), 61.8(\mathrm{C}-6)$, $20.5\left(3 \mathrm{COCH}_{3}\right) ;{ }^{1} \mathrm{H}, \delta 8.639\left(\mathrm{~s}, 1 \mathrm{H}, \mathrm{OCNHCCl}_{3}\right), 6.570(\mathrm{~d}, 1 \mathrm{H}, \mathrm{H}-1), 5.820(\mathrm{~m}, 1 \mathrm{H}$, $\left.\mathrm{H}_{2} \mathrm{C}=\mathrm{CHCH}_{2} \mathrm{O}\right), 5.578(\mathrm{bd}, 1 \mathrm{H}, \mathrm{H}-4), 5.284$ and $5.184(2 \mathrm{~m}$, each $1 \mathrm{H}$, $\mathrm{H}_{2} \mathrm{C}=\mathrm{CHCH}_{2} \mathrm{O}$ ), $5.252(\mathrm{dd}, 1 \mathrm{H}, \mathrm{H}-2), 4.354$ (bt, $\left.1 \mathrm{H}, \mathrm{H}-5\right), 4.206$ (dd, $\left.1 \mathrm{H}, \mathrm{H}-6 \mathrm{a}\right), 4.052$ (dd, $1 \mathrm{H}, \mathrm{H}-6 \mathrm{~b}), 3.947$ (dd, $1 \mathrm{H}, \mathrm{H}-3), 2.160,2.047$, and 2.039 ( $3 \mathrm{~s}$, each $3 \mathrm{H}, 3 \mathrm{Ac}$ ); $J_{1,2} 3.6$, $J_{2,3} 10.4, J_{3.4} 3.4, J_{4,5}<1, J_{5,6 \mathrm{a}} 5.9, J_{5,6 \mathrm{~b}} 7.0, J_{6 \mathrm{a}, 6 \mathrm{~b}}-11.4 \mathrm{~Hz}$.

Anal. Calc. for $\mathrm{C}_{17} \mathrm{H}_{22} \mathrm{Cl}_{3} \mathrm{NO}_{9}: \mathrm{C}, 41.61 ; \mathrm{H}, 4.52$. Found: $\mathrm{C}, 41.01 ; \mathrm{H}, 4.47$.

Ethyl 4-O-(3-O-allyl- $\beta$-D-galactopyranosyl)-2,3-di-O-benzyl-1-thio- $\alpha$-L-rhamnopyranoside (8). - A solution of $5(1.50 \mathrm{~g}, 3.06 \mathrm{mmol})$ and ethyl 2,3-di- $O$-benzyl-1-thio$\alpha$-L-rhamnopyranoside ${ }^{18}(6 ; 0.93 \mathrm{~g}, 2.40 \mathrm{mmol})$ in dry dichloromethane $(15 \mathrm{~mL})$ containing molecular sieves ( $4 \AA, 1 \mathrm{~g}$ ) was stirred for $1 \mathrm{~h}$ under argon. A solution of trimethylsilyl triflate $(28 \mu \mathrm{L})$ in dichloromethane $(0.5 \mathrm{~mL})$ was added at $-30^{\circ}$, and after 5 min, when t.l.c. showed the disappearance of 6 and a new spot $7\left[R_{\mathrm{r}} 0.65,2: 1\right.$ light petroleum (b.p. $40-60^{\circ}$ )-ethyl acetate], pyridine was added, and the mixture was filtered through Celite and concentrated. To a solution of the residue in dry methanol $(25 \mathrm{~mL})$ was added sodium methoxide $(0.99 \mathrm{~g}, 18.0 \mathrm{mmol})$. After $16 \mathrm{~h}$, the solution was neutralised with Dowex-50 $\left(\mathrm{H}^{+}\right)$resin, filtered, and concentrated. Column chromatography ( $8: 2$ dichloromethane-acetone) of the residue gave 8 , isolated as a white glass $(0.99 \mathrm{~g}, 70 \%),[\alpha]_{D}-59^{\circ}$ (c 1, chloroform), $R_{\mathrm{F}} 0.40$ (8:2 dichloromethane-acetone). N.m.r. data $\left(\mathrm{CDCl}_{3}\right):{ }^{13} \mathrm{C}, \delta \quad 137.4-136.8$ and $128.2-127.4\left(\mathrm{C}_{6} \mathrm{H}_{5} \mathrm{CH}_{2} \mathrm{O}\right), 134.3$ $\left(\mathrm{H}_{2} \mathrm{C}=\mathrm{CHCH}_{2} \mathrm{O}\right), 117.4\left(\mathrm{H}_{2} \mathrm{C}=\mathrm{CHCH}_{2} \mathrm{O}\right), 104.6\left(\mathrm{C}-1^{\prime}\right), 81.1,80.1,79.6,78.6,75.6$, $74.0,71.4,67.8$, and $66.1\left(\mathrm{C}-1,2,3,4,5,2^{\prime}, 3^{\prime}, 4^{\prime}, 5^{\prime}\right), 71.6$ and $71.3\left(2 \mathrm{C}_{6} \mathrm{H}_{5} \mathrm{CH}_{2} \mathrm{O}\right), 70.6$ $\left(\mathrm{H}_{2} \mathrm{C}=\mathrm{CHCH}_{2} \mathrm{O}\right), 61.3\left(\mathrm{C}^{-6}\right), 25.1\left(\mathrm{CH}_{3} \mathrm{CH}_{2} \mathrm{~S}\right), 17.3(\mathrm{C}-6), 14.6\left(\mathrm{CH}_{3} \mathrm{CH}_{2} \mathrm{~S}\right) ;{ }^{1} \mathrm{H}, \delta$ $7.365=7.219(\mathrm{~m}, 10 \mathrm{H}, 2 \mathrm{Ph}), 5.936\left(\mathrm{~m}, 1 \mathrm{H}, \mathrm{H}_{2} \mathrm{C}=\mathrm{CHCH}_{2} \mathrm{O}\right), 5.313$ and $5.2 .15(2 \mathrm{~m}$, each $\left.1 \mathrm{H}, \mathrm{H}_{2} \mathrm{C}=\mathrm{CHCH}_{2} \mathrm{O}\right), 5.260(\mathrm{~s}, 1 \mathrm{H}, \mathrm{H}-1), 5.138$ and $4.537(2 \mathrm{~d}$, each $1 \mathrm{H}$, $\left.\mathrm{PhCH}_{2} \mathrm{O}\right), 4.500\left(\mathrm{~s}, 2 \mathrm{H}, \mathrm{PhCH}_{2} \mathrm{O}\right), 4.483\left(\mathrm{~d}, 1 \mathrm{H}, \mathrm{H}-1^{\prime}\right), 4.015(\mathrm{~m}, 1 \mathrm{H}, \mathrm{H}-5), 3.985$ (bd, 1 $\left.\mathrm{H}, \mathrm{H}-4^{\prime}\right), 3.710(\mathrm{t}, 1 \mathrm{H}, \mathrm{H}-4), 3.495\left(\mathrm{bt}, 1 \mathrm{H}, \mathrm{H}-5^{\prime}\right), 3.347\left(\mathrm{dd}, 1 \mathrm{H}, \mathrm{H}-3^{\prime}\right), 2.610=2.507$ (m, $\left.2 \mathrm{H}, \mathrm{CH}_{3} \mathrm{CH}_{2} \mathrm{~S}\right), 1.368(\mathrm{~d}, 3 \mathrm{H}, 3 \mathrm{H}-6), 1.229\left(\mathrm{t}, 3 \mathrm{H}, \mathrm{CH}_{3} \mathrm{CH}_{2} \mathrm{~S}\right) ; J_{1,2} \sim 0, J_{3,4}=J_{4,5}=8.9$, $J_{5,6} 6.2, J_{1^{\prime}, 2^{\prime}} 7.7, J_{2^{\prime}, 3^{\prime}} 9.4, J_{3^{\prime}, 4^{\prime}} 3.4, J_{4^{\prime}, 5^{\prime}}<1, J_{\mathrm{CH}_{2} \mathrm{CH}_{3}} 7.4 \mathrm{~Hz}$. 
Anal. Calc. for $\mathrm{C}_{31} \mathrm{H}_{42} \mathrm{O}_{4} \mathrm{~S} \cdot \mathrm{H}_{2} \mathrm{O}: \mathrm{C}, 61.17$; H. 7.29. Found: C. 61.64; H. 7.08.

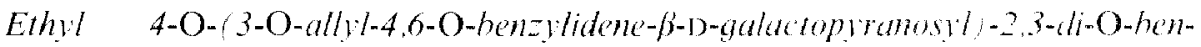
zy-l-thio-x-L-rhamnopyranoside (9). To a solution of $8(990 \mathrm{mg} .1 .68 \mathrm{mmol}$ ) in $N, N$-dimethylformamide $(4 \mathrm{~mL}$ ) and $\alpha$-dimethoxytoluene $(2 \mathrm{~mL}$ ) was added $p$-toluenesulfonic acid $(150 \mathrm{mg})$. After $2 \mathrm{~h}$. t.l.c. indicated the reaction 10 be complete. and solid sodium hydrogencarbonate was added. The mixture wats diluted with dichloromethane $(100 \mathrm{~mL})$. washed with water, dried $\left(\mathrm{Na}_{2} \mathrm{SO}_{4}\right)$. filtered. and concentrated. Column chromatography (95:5 dichloromethane ethyl acetate $)$ of the residue afforded 9, isolated as a syrup $(0.90 \mathrm{~g} .79 \%) .[x]_{1,}-47 \% 1$, chloroform), $R, 0.69(9: 1$ dichloromethane ethyl acetate). N.m.r. data $\left(\mathrm{CDCl}_{3}\right)$ : $\mathrm{C}$. 0137.8137 .0 and 128.7126 .3 $\left(\mathrm{C}_{6} \mathrm{H}_{5} \mathrm{CH}_{2} \mathrm{O}\right.$ and $\left.\mathrm{C}_{6} \mathrm{H}_{5} \mathrm{CH}\right) .134 .9\left(\mathrm{H}_{2} \mathrm{C}=\mathrm{CHCH}, \mathrm{O}\right), 117.2(\mathrm{H}, \mathrm{C}=\mathrm{CHCHO}), 104.6$ $\left(\mathrm{C}^{-1}\right), 100.9$ (PhCH), 81.4, 79.9, 78.7, 78.5, 75.9. 73.2, 70.9, 68.0. and 66.4 $(\mathrm{C}-1,2,3,4,5,2,3,4,5), 71.8$ and $71.6(2 \mathrm{PhCH}, 0), 70.5(\mathrm{HC}=\mathrm{CHCH}, 0), 69.1(\mathrm{C}-6)$. $25.2\left(\mathrm{CH}_{3} \mathrm{CH}_{2} \mathrm{~S}\right), 17.5(\mathrm{C}-6) .14 .8\left(\mathrm{CH}_{3} \mathrm{CH}_{2} \mathrm{~S}\right) ;{ }^{1} \mathrm{H} .67 .4997 .252(\mathrm{~m}, 15 \mathrm{H}, 3 \mathrm{Ph}) .5 .959$ (m. $\left.1 \mathrm{H}, \mathrm{H}_{2} \mathrm{C}=\mathrm{CHCH}_{2} \mathrm{O}\right) .5 .501(\mathrm{~s}, 1 \mathrm{H} . \mathrm{PhCH}) .5 .308$ and $4.693(2 \mathrm{~m}$. each $1 \mathrm{H}$. $\left.\mathrm{H}_{2} \mathrm{C}=\mathrm{CHCH}_{2} \mathrm{O}\right), 5.261(\mathrm{~d}, 1 \mathrm{H} . \mathrm{H}-1) .4 .646,4.562(2 \mathrm{H})$ and $4.515(3 \mathrm{~d} .1,2$ and $1 \mathrm{H} .2$ $\left.\mathrm{PhCH}_{2} \mathrm{O}\right), 4.571(\mathrm{~d}, 1 \mathrm{H}, \mathrm{H}-1) .4 .266$ and $4.023(2 \mathrm{~m}$, each $\left.1 \mathrm{H}, \mathrm{H}, \mathrm{C}=\mathrm{CHCH}, \mathrm{O}), 4.22\right)$ (d, 2 H. 2 H-6). 4.186(bd, 1 H.H.4), $4.054(\mathrm{~m}, 1 \mathrm{H}, \mathrm{H}-5) .3 .922(\mathrm{dd} .1 \mathrm{H}, \mathrm{H}-2) .3 .880(\mathrm{t}$. $1 \mathrm{H}, \mathrm{H}-4), 3.826(\mathrm{dd}, 1 \mathrm{H}, \mathrm{H}-3), 3.789(\mathrm{dd}, 1 \mathrm{H}, \mathrm{H}-2), 3.434(\mathrm{dd}, 1 \mathrm{H}, \mathrm{H}-3), 3.370(\mathrm{bs}, 1 \mathrm{H}$, $\left.\mathrm{H}-5^{\prime}\right) .2 .607 \cdots 2.501\left(\mathrm{~m}, 2 \mathrm{H}, \mathrm{CH}_{3} \mathrm{CH} \mathrm{S}\right), 1.402(\mathrm{~d}, 3 \mathrm{H}, 3 \mathrm{H}-6) 1.226(1,3 \mathrm{H}, \mathrm{CH}, \mathrm{CH} \mathrm{S})$.

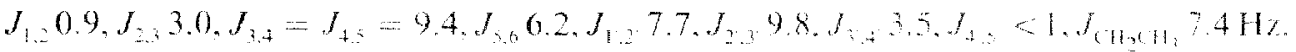
Anal. Calc. for $\mathrm{C}_{38} \mathrm{H}_{4 i}, \mathrm{O}, \mathrm{S}: \mathrm{C}$. 67.24; H. 6.83. Found: C. 67.06: 11. 0.92.

2,3,4-Tri-O-acety-x-1-shamnopyranosy trichloroacetimidate (10). To at solution of I-rhamnose tetra-acetale (19.33 g. $58.22 \mathrm{mmol})$ in dry $\mathrm{V}$. V-dimethylformamide $(50 \mathrm{~mL})$ was added hydrazine acetate $(5.99 \mathrm{~g}, 65.04 \mathrm{mmol})$. After 1 h. ethylacetate $(600)$ $\mathrm{mL}$ ) was added, and the organic phase was washed with aqueous $5 \%$ " sodium chloride and water, dried $\left(\mathrm{Na}_{2} \mathrm{SO}_{4}\right)$. filtered. and concentrated to gite 2.3.4-1ri-O-acetyl- $\not, 3-1-$ rhamnopyranose, isolated as a white foam $(16.53 \mathrm{~g}, 98 \% \mathrm{n}), R_{1}, 0.1619 .1$ dichloromethane ethyl acetate). To at solution of part $(4.00 \mathrm{~g}, 13.79 \mathrm{mmol})$ of the product in dichloromethane $(170 \mathrm{~mL})$ and trichloroacetonitrile $(17.0 \mathrm{~mL} .169 .5 \mathrm{mmol})$ was added a solution of 1.8-diazabicyclo[5.4.0]undec-7-ene $(2.06 \mathrm{~mL} .13 .79 \mathrm{mmol})$ in dichloromethane $(20 \mathrm{~mL})$ at 0 . The mixture was stirred for 1 h at room temperature, when the reaction was complete (t.l.c), and then concentrated. Column chromatography $(9: 1$ dichloromethane ethyl acetate of the residue gave 10. isolated as a yellow syup $5.36 \mathrm{~g}$. $90 \%),[x]_{1},-52$ (a 1 , chlorofom), $R_{1} 0.73$ (9:1 dichloromethane chy acetate). N.m. data $\left(\mathrm{CDCl}_{3}\right):{ }^{17} \mathrm{C}, \delta 169.7\left(\mathrm{COCH}_{3}\right), 94.7(\mathrm{C}-1), 70.3,69.2,68.7$. and $68.1(\mathrm{C}-2.3 .4 .5)$. $17.4(\mathrm{C}-6), 20.6(2 \mathrm{C})$, and $20.5\left(\mathrm{COCH}_{3}\right) ; \mathrm{H}, 8.734(\mathrm{~s}, 1 \mathrm{H} . \mathrm{OCNHCCl}), 6.204(\mathrm{~d} .1 \mathrm{H}$. $\mathrm{H}-1) .5 .463$ (dd, $1 \mathrm{H}, \mathrm{H}-2), 5.370(\mathrm{dd}, 1 \mathrm{H}, \mathrm{H}-3), 5.179(\mathrm{t}, 1 \mathrm{H}, \mathrm{H}-4) .4093(\mathrm{~m}, 1 \mathrm{H}, \mathrm{H}-5)$.

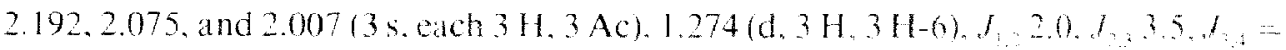
$J_{4.5}=10.2, J_{5,6} 6.3 \mathrm{~Hz}$

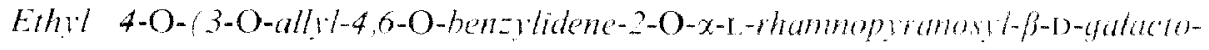
pyranosyl -2,3-di-O-henz-1-1-thio-x-1-rhammoplranoside (12). . To a solution of 9 $(0.90 \mathrm{~g} .1 .33 \mathrm{mmol})$ and $10(0.85 \mathrm{~g} .1 .96 \mathrm{mmol})$ in dry dichoromethane (10 wL) 
containing powdered molecular sieves $(4 \AA, 0.5 \mathrm{~g})$ was added a solution of trimethylsilyl triflate $(8 \mu \mathrm{L})$ in dichloromethane $(0.5 \mathrm{~mL})$ at $-30^{\circ}$. After $5 \mathrm{~min}$, t.l.c. $(9: 1$ dichloromethane-ethyl acetate) showed the disappearance of $9\left(R_{\mathrm{r}} 0.69\right)$ and a new compound at $R_{\mathrm{F}} 0.84$ (11). Pyridine $(0.2 \mathrm{~mL})$ was added, the mixture was filtered and concentrated, and the residue was deacetylated with sodium methoxide in methanol $(\mathrm{pH} 10)$ for $16 \mathrm{~h}$. The solvent was evaporated, and a solution of the residue in dichloromethane $(250 \mathrm{~mL})$ was washed with water, dried $\left(\mathrm{Na}_{2} \mathrm{SO}_{4}\right)$, filtered, and concentrated. Column chromatography (7:3 dichloromethane-acetone) of the residue gave 12, isolated as a white glass $(0.85 \mathrm{~g}, 78 \%),[\alpha]_{\mathrm{V}}-69^{\circ}$ ( $\mathrm{c} 1$, chloroform), $R_{\mathrm{F}} 0.62$ (7:3 dichloromethane-acetone). N.m.r. data $\left(\mathrm{CDCl}_{3}\right):{ }^{13} \mathrm{C}, \delta 138.3-137.7$ and $128.9-126.4\left(C_{6} \mathrm{H}_{5} \mathrm{CH}_{2} \mathrm{O}\right.$ and $\left.C_{6} \mathrm{H}_{5} \mathrm{CH}\right)$, $134.6\left(\mathrm{H}_{2} \mathrm{C}=\mathrm{CHCH}_{2} \mathrm{O}\right), 117.4\left(\mathrm{H}_{2} \mathrm{C}=\mathrm{CHCH}_{2} \mathrm{O}\right), 101.1,100.7$, and $100.6\left(\mathrm{C}-1^{\prime}, 1^{\prime \prime}\right.$, and $\mathrm{PhCH}$ ), 81.3, 80.3, 80.0, 75.5 (2 C), 75.3, 73.3, 72.9, 71.7, 70.6, 67.9, 67.7, and 65.7 $\left(\mathrm{C}-1,2,3,4,5,2^{\prime}, 3^{\prime}, 4^{\prime}, 5^{\prime}, 2^{\prime \prime}, 3^{\prime \prime}, 4^{\prime \prime}, 5^{\prime \prime}\right), 71.6$ and $71.3\left(2 \mathrm{PhCH}_{2} \mathrm{O}\right), 70.3\left(\mathrm{H}_{2} \mathrm{C}=\mathrm{CHCH}_{2} \mathrm{O}\right)$, $69.1\left(\mathrm{C}-6^{\prime}\right), 25.3\left(\mathrm{CH}_{3} \mathrm{CH}_{2} \mathrm{~S}\right), 17.9$ and $17.1\left(\mathrm{C}-6,6^{\prime \prime}\right), 14.9\left(\mathrm{CH}_{3} \mathrm{CH}_{2} \mathrm{~S}\right) ;{ }^{1} \mathrm{H}, \delta 7.505-7.256$ $(\mathrm{m}, 15 \mathrm{H}, 3 \mathrm{Ph}), 5.915\left(\mathrm{~m}, 1 \mathrm{H}, \mathrm{H}_{2} \mathrm{C}=\mathrm{CHCH}_{2} \mathrm{O}\right), 5.475(\mathrm{~s}, 1 \mathrm{H}, \mathrm{PhCH}), 5.301$ and 5.215 $\left(2 \mathrm{~m}\right.$, each $\left.1 \mathrm{H}, \mathrm{H}_{2} \mathrm{C}=\mathrm{CHCH}_{2} \mathrm{O}\right), 5.301$ (bs, $\left.1 \mathrm{H}, \mathrm{H}-1\right), 5.148\left(\mathrm{~d}, 1 \mathrm{H}, \mathrm{H}-\mathrm{l}^{\prime \prime}\right), 4.899(\mathrm{~d}, 1$ $\mathrm{H}, \mathrm{H}-\mathrm{l}^{\prime}$ ), 4.657, 4.595, 4.572, and 4.442 (4 d, each $1 \mathrm{H}, 2 \mathrm{PhCH}_{2} \mathrm{O}$ ), 4.168 (bd, $1 \mathrm{H}, \mathrm{H}-4^{\prime}$ ), 3.912 (dd, 1 H, H-2'), 3.439 (dd, 1 H, H-3'), 3.325 (bs, $\left.1 \mathrm{H}, \mathrm{H}-5^{\prime}\right), 2.642-2.538$ (m, 2 H, $\mathrm{CH}_{3} \mathrm{CH} \mathrm{H}_{2} \mathrm{~S}$ ), 1.420 and 1.335 ( $2 \mathrm{~d}$, each $3 \mathrm{H}, 3 \mathrm{H}-6$ and $\left.3 \mathrm{H}-6^{\prime \prime}\right), 1.249\left(\mathbf{t}, 3 \mathrm{H}, \mathrm{CH}_{3} \mathrm{CH}_{2} \mathrm{~S}\right)$, $J_{1,2} \sim 0, J_{5,6,} J_{S^{\prime \prime}, 6^{\prime \prime}} 6.0 / 6.3, J_{1^{\prime}, 2^{\prime}} 7.7, J_{2^{\prime}, 3^{\prime}} 9.8, J_{3^{\prime}, 4^{\prime}} 3.5, J_{4^{\prime}, S^{\prime}}<1, J_{1^{\prime \prime}, 2^{\prime \prime}} 1.3, J_{3^{\prime \prime}, 4^{\prime \prime}}=J_{4^{\prime \prime}, S^{\prime \prime}}=9.5$, $J_{\mathrm{CH}_{2} \mathrm{CH}_{3}} 7.4 \mathrm{~Hz}$.

Anal. Calc. for $\mathrm{C}_{44} \mathrm{H}_{56} \mathrm{O}_{13} \mathrm{~S} \cdot \mathrm{H}_{2} \mathrm{O}: \mathrm{C}, 62.69 ; \mathrm{H}, 6.93$. Found: $\mathrm{C}, 63.10 ; \mathrm{H}, 6.97$.

Ethyl 4-O-[3-O-allyl-4,6-O-benzylidene-2-O-(2,3,4-tri-O-benzyl- $\alpha$-L-rhamnopyranosyl)- $\beta$-D-galactopyranosyl]-2,3-di-O-benzyl-1-thio- $\alpha$-L-rhamnopyranoside (13). A solution of $12(0.43 \mathrm{~g}, 0.52 \mathrm{mmol})$ and benzyl bromide $(0.4 \mathrm{~mL}, 3.3 \mathrm{mmol})$ in dry $N, N$-dimethylformamide $(7 \mathrm{~mL})$ was added to a stirred suspension of sodium hydride $(0.15 \mathrm{~g}, 6.0 \mathrm{mmol})$ in $N, N$-dimethylformamide $(2.5 \mathrm{~mL})$ at $0^{\circ}$. After $2 \mathrm{~h}$, t.l.c. indicated the disappearance of $\mathbf{1 2}$, and methanol was added to destroy the excess of sodium hydride. The mixture was poured into ice-water $(100 \mathrm{~mL})$ and extracted with ether $(3 \times$ $30 \mathrm{~mL})$, and the combined extracts were dried $\left(\mathrm{Na}_{2} \mathrm{SO}_{4}\right)$, filtered, and concentrated. Column chromatography (95:5 dichloromethane-ethyl acetate) of the residue gave 13, isolated as a syrup $(0.53 \mathrm{~g}, 93 \%),\lfloor\alpha\rfloor_{\mathrm{D}}-40^{\circ}$ (c 1 , chloroform), $R_{\mathrm{r}} 0.62$ (95:5 dichloromethane-ethyl acetate). N.m.r. data $\left(\mathrm{CDCl}_{3}\right):{ }^{13} \mathrm{C}, \delta 139.2-137.8$ and $128.9-126.5$ $\left(\mathrm{C}_{6} \mathrm{H}_{5} \mathrm{CH}_{2} \mathrm{O}\right.$ and $\left.\mathrm{C}_{6} \mathrm{H}_{5} \mathrm{CH}\right), 134.8\left(\mathrm{H}_{2} \mathrm{C}=\mathrm{CHCH}_{2} \mathrm{O}\right), 117.2\left(\mathrm{H}_{2} \mathrm{C}=\mathrm{CHCH}_{2} \mathrm{O}\right), 101.2$ and $100.7\left(\mathrm{C}-1^{\prime}\right.$ and $\left.\mathrm{PhCH}\right), 98.8\left(\mathrm{C}-1^{\prime \prime}\right), 81.3,80.5,80.4,80.3,79.9,75.6,75.5,75.0,74.6$, $72.9,67.9,67.7$, and $65.7\left(\mathrm{C}-1,2,3,4,5,2^{\prime}, 3^{\prime}, 4^{\prime}, 5^{\prime}, 2^{\prime \prime}, 3^{\prime \prime}, 4^{\prime \prime}, 5^{\prime \prime}\right), 74.7,72.0(2 \mathrm{C}), 71.6$, and $71.0\left(5 \mathrm{PhCH}_{2} \mathrm{O}\right), 70.1\left(\mathrm{H}_{2} \mathrm{C}=\mathrm{CHCH}_{2} \mathrm{O}\right), 69.2\left(\mathrm{C}-6^{\prime}\right), 25.3\left(\mathrm{CH}_{3} \mathrm{CH}_{2} \mathrm{~S}\right), 18.0$ and 17.6 $\left(\mathrm{C}-6,6^{\prime \prime}\right), 15.0\left(\mathrm{CH}_{3} \mathrm{CH}_{2} \mathrm{~S}\right) ;{ }^{1} \mathrm{H}, \delta 7.363-7.178(\mathrm{~m}, 30 \mathrm{H}, 6 \mathrm{Ph}), 5.765(\mathrm{~m}, 1 \mathrm{H}$, $\left.\mathrm{H}_{2} \mathrm{C}=\mathrm{C} H \mathrm{CH}_{2} \mathrm{O}\right), 5.473(\mathrm{~s}, 1 \mathrm{H}, \mathrm{PhCH}), 5.283(\mathrm{bs}, 1 \mathrm{H}, \mathrm{H}-1), 5.262$ and $5.150(2 \mathrm{~m}$, each $\left.1 \mathrm{H}, \mathrm{H}_{2} \mathrm{C}=\mathrm{CHCH}_{2} \mathrm{O}\right), 5.217\left(\mathrm{~d}, 1 \mathrm{H}, \mathrm{H}-\mathrm{1}^{\prime \prime}\right), 4.905\left(\mathrm{~d}, 1 \mathrm{H}, \mathrm{H}-1^{\prime}\right), 4.383\left(\mathrm{~m}, 1 \mathrm{H}, \mathrm{H}-5^{\prime \prime}\right)$, $3.587\left(\mathrm{t}, 1 \mathrm{H}, \mathrm{H}-4^{\prime \prime}\right), 3.431$ (dd, $\left.1 \mathrm{H}, \mathrm{H}-3^{\prime}\right), 3.336\left(\mathrm{bs}, 1 \mathrm{H}, \mathrm{H}-5^{\prime}\right), 2.658-2.525(\mathrm{~m}, 2 \mathrm{H}$, $\left.\mathrm{CH}_{3} \mathrm{CH}_{2} \mathrm{~S}\right), 1.423\left(\mathrm{~d}, 6 \mathrm{H}, 3 \mathrm{H}-6\right.$ and $\left.3 \mathrm{H}-6^{\prime \prime}\right), 1.259\left(\mathrm{t}, 3 \mathrm{H}, \mathrm{CH}_{3} \mathrm{CH}_{2} \mathrm{~S}\right), J_{1,2} \sim 0, J_{5,6} / J_{5^{\prime \prime}, 6^{\prime \prime}}$ $6.2 / 6.2, J_{1^{\prime}, 2^{\prime}} 7.7, J_{2^{\prime}, 3^{\prime}} 9.8, J_{3^{\prime}, 4^{\prime}} 3.5, J_{4^{\prime} 5^{\prime}}<1, J_{1^{\prime \prime}, 2^{\prime}} 1.4, J_{3^{\prime \prime}, 4^{\prime \prime}}=J_{4^{\prime \prime}, 5^{\prime \prime}}=9.5, J_{\mathrm{CH}_{2} \mathrm{CH}_{3}} 7.4 \mathrm{~Hz}$. 
Anal. Calc. for $\mathrm{C}_{65} \mathrm{H}_{74} \mathrm{O}_{13} \mathrm{~S}: \mathrm{C}, 71.28 ; \mathrm{H}, 6.81$. Found: $\mathrm{C}, 71.17 ; \mathrm{H} .6 .99$.

Ethyl 4-O-13-O-allyl-6-O-benzy-2-O-12,3,4-tri-O-benzy-x-L - hammop!rano-

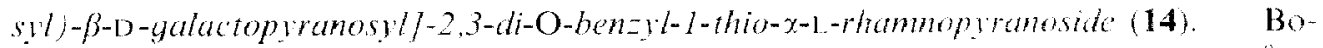
rane trimethylamine complex ${ }^{2 *}(0.36 \mathrm{~g}, 4.93 \mathrm{mmol})$, powdered molecular sieves $(4 \AA .2$ g), and $13(0.47 \mathrm{~g}, 0.43 \mathrm{mmol})$, in tetrahydrofuran $(20 \mathrm{ml})$ were stired for $1 \mathrm{~h}$. Aluminium(III) chloride $(0.79 \mathrm{~g}, 5.94 \mathrm{mmol})$ was added at 0 and the mixture was stirred overnight at room temperature. T.l.c. (9:1 dichloromethane ethylacelate) then showed the conversion of $13\left(R_{\mathrm{F}} 0.82\right)$ into $14(R, 0.43)$. The mixture was filtered, washed with cold $0.5 \mathrm{~m}$ sulfuric acid. water, aqueous $5 \%$ sodium hydrogencarbonate. and water. dried $\left(\mathrm{Na}_{2} \mathrm{SO}_{4}\right)$, filtered, and concentrated. Column chromatography $(9,1$ dichloromethane ethyl acetate) of the residue afforded 14 . isolated as a syrup $\left(0,32\right.$ g. $\left.69^{\circ} \%\right),[x]$, -54 (c 1, chloroform). N.m.r. data $\left(\mathrm{CDCl}_{3}\right):{ }^{7} \mathrm{C} . \delta 139.1 \% 137.7$ and 128.2127 .0 $\left(\mathrm{C}_{6} \mathrm{H}_{5} \mathrm{CH}_{2} \mathrm{O}\right), 134.2\left(\mathrm{H}_{2} \mathrm{C}=\mathrm{CHCH}_{2} \mathrm{O}\right), 117.4\left(\mathrm{H}_{2} \mathrm{C}=\mathrm{CHCH}_{2} \mathrm{O}\right) 101.0(\mathrm{C}-1), 98.8$ $\left(\mathrm{C}-1 "^{\prime \prime}\right), 25.2\left(\mathrm{CH}_{3} \mathrm{CH}, \mathrm{S}\right), 17.8$ and $17.5\left(\mathrm{C}-6,6^{\prime \prime}\right), 14.9\left(\mathrm{CH}_{3} \mathrm{CH}_{2} \mathrm{~S}\right):{ }^{3} \mathrm{H} .7 .3437 .064(\mathrm{~m}$. $30 \mathrm{H}, 6 \mathrm{Ph}), 5.727\left(\mathrm{~m} .1 \mathrm{H} . \mathrm{H}, \mathrm{C}=\mathrm{CHCH}_{2} \mathrm{O}\right), 5.250(\mathrm{~d}, 1 \mathrm{H}, \mathrm{H}-1), 5205$ (d, I H. H-l") 5.263 and $5.164\left(2 \mathrm{~m}\right.$, ach $\left.\left.1 \mathrm{H}, H_{2} \mathrm{C}=\mathrm{CHCH}_{2} \mathrm{O}\right) .4856(\mathrm{~d}) \mathrm{H}, \mathrm{H}-\mathrm{I}^{\prime}\right) .4 .343(\mathrm{~m} . \mathrm{H} \mathrm{H}$. $\left.H-5^{\prime \prime}\right), 4.006(\mathrm{~m}, 1 \mathrm{H}, \mathrm{H}-5) .3 .370(\mathrm{dd} .1 \mathrm{H}, \mathrm{H}-3) .2 .623-2.530\left(\mathrm{~m} .2 \mathrm{H} . \mathrm{CH}_{3} \mathrm{CH}_{-} \mathrm{S}\right) .1 .386$

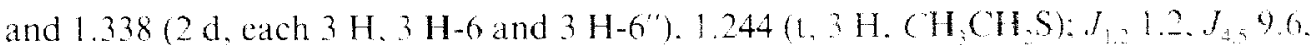

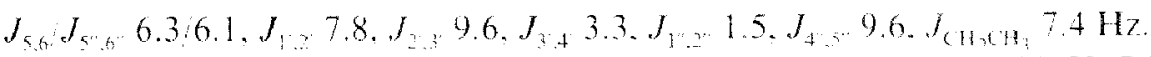

Anal. Calc. for $\mathrm{C}_{65} \mathrm{H}_{76} \mathrm{O}_{13} \mathrm{~S}: \mathrm{C}, 71.14 ; \mathrm{H}, 6.98$. Found: C.71.01: H. 7.05

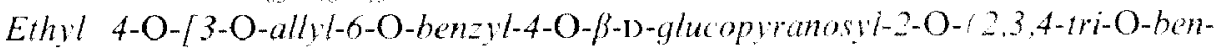

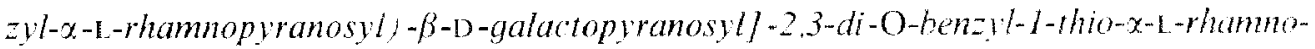
pyranoside (17). To a solution of $14(0.65 \mathrm{~g}, 0.59 \mathrm{mmol})$ and $2.3 .4,6$-letra-O-acetyl- - D-glucopyranosyl trichloroacetimidate ${ }^{29}(15 ; 0.40 \mathrm{~g}, 0.81 \mathrm{mmol})$ in dry dichloromethane $(10 \mathrm{~mL})$ containing powdered molecular sieves $(4 \AA, 0.50 \mathrm{~g})$ was added a solution of trimethylsilyl triflate $(14 \mu \mathrm{L})$ in dichloromethane $(1 \mathrm{~mL})$ at -10$)$. As shown by t.lc. $(9: 1$ dichloromethane-ethyl acetate), $\mathbf{1 6}(R, 0.55)$ was formed in $5 \mathrm{~min}$. Pyridine was added. and the mixture was filtered and concentrated. Conventional deacetylation and column chromatography $(65: 35$ dichloromethane-acetone $)$ of the product afforded 17 , isolated as a syrup $(0.50 \mathrm{~g}, 67 \%),[x]_{\mathrm{L}}-39 \%\left(c 1\right.$, chloroform), $R_{,} 0.50(65: 35$ dichloromethane acetone). N.m.r. data $\left(\mathrm{CDCl}_{3}\right):{ }^{17} \mathrm{C} . \delta 139.0-137.7$ and $128.4127 .0\left(\mathrm{C}_{4} \mathrm{H}_{5} \mathrm{CH}_{2} \mathrm{O}\right) .133 .4$ $\left(\mathrm{H}_{2} \mathrm{C}=\mathrm{CHCH}_{2} \mathrm{O}\right), 118.6\left(\mathrm{H}_{2} \mathrm{C}=\mathrm{CHCH}_{2} \mathrm{O}\right), 105.5(\mathrm{C}-1 " \cdots), 1010(\mathrm{C}-1), 98.9(\mathrm{C}-1 ")$, $25.3\left(\mathrm{CH}_{3} \mathrm{CH}_{2} \mathrm{~S}\right), 17.9$ and $17.4\left(\mathrm{C}-6,6^{\prime \prime}\right), 14.9\left(\mathrm{CH}_{3} \mathrm{CH}_{2} \mathrm{~S}\right) ; \mathrm{H}, 7.389-7.066(\mathrm{~m}, 30 \mathrm{H}, 6$ $\mathrm{Ph}), 5.701\left(\mathrm{~m}, 1 \mathrm{H}, \mathrm{H}_{2} \mathrm{C}=\mathrm{CHCH}_{2} \mathrm{O}\right), 5.261$ and $5.196\left(2 \mathrm{~m}\right.$, each $\left.1 \mathrm{H}_{1} \mathrm{H}_{2} \mathrm{C}=\mathrm{CHCH}_{2} \mathrm{O}\right)$. 5.256 (d, $1 \mathrm{H}, \mathrm{H}-1), 5.131$ (d, $\left.1 \mathrm{H}, \mathrm{H}-\mathrm{I}^{\prime \prime}\right), 4.821$ (d, $\left.1 \mathrm{H} . \mathrm{H}-\mathrm{l}^{\prime}\right) .4 .311$ (d. $\left.1 \mathrm{H}, \mathrm{H}-\mathrm{I}^{\prime \prime}\right)$, $2.627-2.526\left(\mathrm{~m}, 2 \mathrm{H}, \mathrm{CH}_{3} \mathrm{CH}_{2} \mathrm{~S}\right), 1.386$ and $1.305\left(2 \mathrm{~d}\right.$, each $3 \mathrm{H}, 3 \mathrm{H} 6$ and $\left.3 \mathrm{H}-6^{\prime \prime}\right), 1.248$

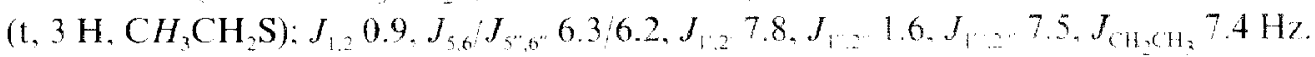

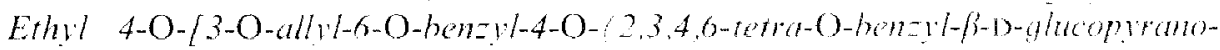

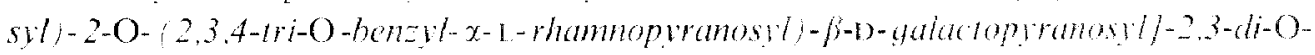
henzy-1-thio- $x-1$-rhammoplraneside (18). A solution of $17(0.50 \mathrm{~g} .0 .40 \mathrm{mmol})$ and benzyl bromide $(0.38 \mathrm{~mL}, 3.2 \mathrm{mmol})$ in $N, N$-dimethylformamide $(5 \mathrm{~mL})$ was added to a stirred suspension of sodium hydride $(0.1 \mathrm{~g}, 4.2 \mathrm{mmol})$ in dry $N$. N-dimethylformamide $(3 \mathrm{~mL})$ at 0 . After $2 \mathrm{~h}$ af ronm temperature, t.l.c. indicated the complete disappearance 
of 17. Methanol was added to destroy the excess of sodium hydride, the mixture was poured into ice-water $(75 \mathrm{~mL})$ and extracted with ether $(3 \times 25 \mathrm{~mL})$, and the combined extracts were dried $\left(\mathrm{Na}_{2} \mathrm{SO}_{4}\right)$, filtered, and concentrated. Column chromatography (97:3 dichloromethane ethyl acetate) of the residue yielded 18 , isolated as a syrup $(0.44$ $\mathrm{g}, 68 \%),[\alpha]_{\mathrm{D}}-31^{\circ}\left(\mathrm{c} 1\right.$, chloroform), $R_{\mathrm{p}} 0.80$ (95:5 dichloromethane ethyl acetate). ${ }^{13} \mathrm{C}-\mathrm{N} . \mathrm{m} . \mathrm{r}$. data $\left(\mathrm{CDCl}_{3}\right): \delta \quad 139.3-137.9$ and $128.9-127.0 \quad\left(\mathrm{C}_{6} \mathrm{H}_{5} \mathrm{CH}_{2} \mathrm{O}\right), 134.3$ $\left(\mathrm{H}_{2} \mathrm{C}=\mathrm{CHCH}_{2} \mathrm{O}\right), 118.6\left(\mathrm{H}, \mathrm{C}=\mathrm{CHCH}_{2} \mathrm{O}\right), 101.6\left(\mathrm{C}-1^{\prime \prime \prime}\right), 101.0\left(\mathrm{C}-1^{\prime}\right), 98.6\left(\mathrm{C}-1^{\prime \prime}\right)$, $25.3\left(\mathrm{CH}_{3} \mathrm{CH}_{2} \mathrm{~S}\right), 17.7\left(\mathrm{C}-6,6^{\prime \prime}\right), 15.0\left(\mathrm{CH}_{3} \mathrm{CH}_{2} \mathrm{~S}\right)$.

Anal. Calc. for $\mathrm{C}_{99} \mathrm{H}_{110} \mathrm{O}_{18} \mathrm{~S}: \mathrm{C}, 73.40 ; \mathrm{H}, 6.84$. Found: C, 73.20; H, 6.99.

Ethyl 2,3-di-O-benzyl-4-O-/6-O-benzyl-4-O- (2,3,4,6-tetra-O-benzyl- $\beta$-D-glucopyranosyl) -2-O-(2,3,4-tri-O-benzyl- $\alpha$-L-rhamnopyranosyl)- $\beta$-D-galactopyranosyl $]-1$ thio- $x$-L-rhamnopyranoside (19). - To a mixture of $18(0.20 \mathrm{~g}, 0.12 \mathrm{mmol})$ and 1,4 diazabicyclo[2.2.2] octane $(103 \mathrm{mg})$ in $8: 3: 1$ ethanol-toluene-water $(6 \mathrm{~mL})$ was added tris(triphenylphosphinc)rhodium(I) chloride $(25 \mathrm{mg})$, and the mix ture was boiled under reflux for $16 \mathrm{~h}$, then cooled, and concentrated. The residue was diluted with dichloromethane $(200 \mathrm{~mL})$, washed with water, cold $\mathrm{m}$ hydrochloric acid $(40 \mathrm{~mL})$, aqueous $5 \%$ sodium hydrogencarbonate, and water, dried $\left(\mathrm{Na}_{2} \mathrm{SO}_{4}\right)$, filtered, and concentrated. $\mathrm{A}$ solution of the residue in acetone $(4.5 \mathrm{~mL})$ and $\mathrm{M}$ hydrochloric acid $(0.5 \mathrm{~mL})$ was boiled under reflux for $2 \mathrm{~h}$, when t.l.c. (95:5 dichloromethane-ethyl acetate) showed the formation of $19\left(R_{\mathrm{F}}, 0.35\right)$. The mixture was neutralised with aqueous $5 \%$ sodium hydrogencarbonate, concentrated, and diluted with dichloromethane $(200 \mathrm{~mL})$, washed with water, dried $\left(\mathrm{Na}_{2} \mathrm{SO}_{4}\right)$, filtered, and concentrated. Column chromatography $(92: 8$ dichloromethane-ethyl acetate) of the residue gave pure 19, isolated as a syrup $(0.14 \mathrm{~g}$, $73 \%),[\alpha]_{\mathrm{D}}-29^{\circ}$ (c 0.6, chloroform). N.m.r. data $\left(\mathrm{CDCl}_{3}\right):{ }^{13} \mathrm{C}, \delta 139.3-137.0$ and 128.5 126.9 ( $\left.\mathrm{C}_{6} \mathrm{H}_{5} \mathrm{CH}_{2} \mathrm{O}\right), 104.4\left(\mathrm{C}-1^{\prime \prime \prime}\right), 100.9\left(\mathrm{C}-1^{\prime}\right), 97.8\left(\mathrm{C}-1^{\prime \prime}\right), 25.3\left(\mathrm{CH}_{3} \mathrm{CH}_{2} \mathrm{~S}\right), 18.0$ and $17.7\left(\mathrm{C}-6,6^{\prime \prime}\right), 15.0\left(\mathrm{CH}_{3} \mathrm{CH}_{2} \mathrm{~S}\right) ;{ }^{1} \mathrm{H}, \delta 7.375-7.021(\mathrm{~m}, 50 \mathrm{H}, 10 \mathrm{Ph}), 5.248(\mathrm{~d}, 1 \mathrm{H}$, $\mathrm{H}-1), 5.115$ (d, $\left.1 \mathrm{H}, \mathrm{H}-1^{\prime \prime}\right), 2.625-2.516\left(\mathrm{~m}, 2 \mathrm{H}, \mathrm{CH}_{3} \mathrm{CH}_{2} \mathrm{~S}\right), 1.395$ and 1.371 (2 d, each 3 H, $3 \mathrm{H}-6$ and $3 \mathrm{H}-6^{\prime \prime}$ ), 1.255 (t, $\left.3 \mathrm{H}, \mathrm{CH}_{3} \mathrm{CH}_{2} \mathrm{~S}\right) ; J_{1,2}<1, J_{5,6} / J_{5^{\prime \prime}, 6^{\prime \prime}} 6.3 / 5.9, J_{1^{\prime \prime}, 2^{\prime \prime}}<1$, $J_{\mathrm{CH}_{2} \mathrm{CH}_{3}} 7.4 \mathrm{~Hz}$.

Ethyl 4-O-[3-O-acetyl-6-O-benzyl-4-O-(2,3,4,6-tetra-O-benzyl- $\beta$-D-glucopyranosyl) -2-O-(2,3,4-tri-O-benzyl- $\alpha$-L -rhamnopyranosyl) - $\beta$ - D-galactopyranosyl]-2,3-di-Obenzyl-1-thio- $\boldsymbol{x}$-L-rhamnopyranoside (20). - A solution of $19(0.13 \mathrm{~g}, 0.08 \mathrm{mmol})$ in pyridine $(2 \mathrm{~mL})$ and acetic anhydride $(2 \mathrm{~mL})$ was stirred overnight, then concentrated, and co-concentrated with toluene $(3 \times 20 \mathrm{~mL})$, ethanol $(3 \times 20 \mathrm{~mL})$, and dichloromethane $(3 \times 20 \mathrm{~mL})$. Column chromatography $(95: 5$ dichloromethane-ethyl acetate $)$ of the residue yielded 20 , isolated as a syrup $(0.13 \mathrm{~g}, 93 \%),[\alpha]_{\mathrm{D}}-24^{\circ}(c 1$, chloroform), $R_{\mathrm{r}} 0.69$ (95:5 dichloromethane-ethyl acetate). ${ }^{13} \mathrm{C}-$ N.m.r. data $\left(\mathrm{CDCl}_{3}\right): \delta 170.1$ $\left(\mathrm{COCH}_{3}\right), 138.6-137.9$ and $128.2-127.0\left(\mathrm{C}_{6} \mathrm{H}_{5} \mathrm{CH}_{2} \mathrm{O}\right), 103.3\left(\mathrm{C}-1^{\prime \prime \prime}\right), 100.9\left(\mathrm{C}-1^{\prime}\right), 98.7$ $\left(\mathrm{C}-1^{\prime \prime}\right), 25.3\left(\mathrm{CH}_{3} \mathrm{CH}_{2} \mathrm{~S}\right), 20.7\left(\mathrm{COCH}_{3}\right), 17.8\left(\mathrm{C}-6,6^{\prime \prime}\right), 15.0\left(\mathrm{CH}_{3} \mathrm{CH}_{2} \mathrm{~S}\right)$.

Anal. Calc. for $\mathrm{C}_{98} \mathrm{H}_{108} \mathrm{O}_{19} \mathrm{~S}: \mathrm{C}, 72.57 ; \mathrm{H}, 6.71$. Found: C, 72.48; H, 6.79.

3-Benzyloxycarbonylaminopropyl 4-O-[3-O-acetyl-6-O-benzyl-4-O-(2,3,4,6-tetra-O-benzyl- $\beta$-D-glucopyranosyl)-2-O-(2,3,4-tri-O-benz $y$ l- $\alpha$-L-rhamnopyranosyl)- $\beta$-D-

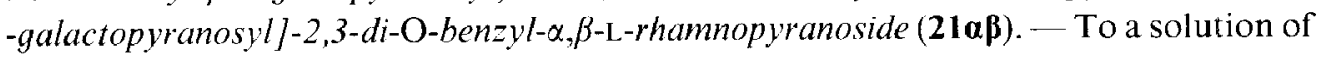


$20(60 \mathrm{mg}, 37 \mu \mathrm{mol})$ in ary dichloromethane $(5 \mathrm{~mL})$ was added a solution of bromine $(20$ $\mu$ L. $0.4 \mathrm{mmol})$ in dry dichloromethane $(0.5 \mathrm{~mL}$ ). After $10 \mathrm{~min}$, the mixture was co-concentrated with dy toluene $(3 \times 5 \mathrm{~mL})$. A solution of the resulting glycosyl bromide in toluene $(1 \mathrm{~mL}$ ) was added to a stirred mixture of 3-benzyloxycarbonylamino-1-propanol ${ }^{32}(80 \mathrm{mg} .0 .41 \mathrm{mmol})$, molecular sieves $(4 \AA ., 100 \mathrm{mg}$ ), and silver silicate (90) $\mathrm{mg}$ ) in dry dichloromethane $(5 \mathrm{~mL}$. After $2 \mathrm{~h}, 1.1 .6 .59$ dichloromethane ethyl acetate) indicated a new slower-moving spot $\left(R_{2}, 0.15\right)$. The mixture was diluted with dichloromethane $(75 \mathrm{~mL}$ ), filtered through Celite, and concentrated Column chromatography (92:8 dichloromethane ethyl acetate) of the residue yelded a mixture of $21 \alpha \beta$ and its analogue with HO-I unsubstituted. After conventional acetylion and column chromatography (92:8 dichloromethane ethyl acetate), $21 \alpha \beta$ (40) $\mathrm{mg}$, 64\% (3) was obtained as a syrup. Nim.s. data $\left.1 \mathrm{CDCl}_{3}\right)$ " $\mathrm{C}$ o $170.0 \quad(\mathrm{COCH}) .56 .3$ $\left(\mathrm{NHCOOCH}_{2} \mathrm{C}_{6} \mathrm{H}_{3}\right), 139.1 \quad 136.6$ and $128.2126 .9\left(\mathrm{C}_{6} \mathrm{H} . \mathrm{CH} \mathrm{O}\right), 103.4\left(\mathrm{C}-\mathrm{l}^{\prime \prime}\right), 101.4$ $(\mathrm{C}-1 \beta), \quad 100.7 \quad(\mathrm{C}-1), 98.7 \quad(\mathrm{C}-1 x), \quad 98.2 \quad(\mathrm{C}-1 \%), 66.4 .65 .3,38.2$, and 29.6

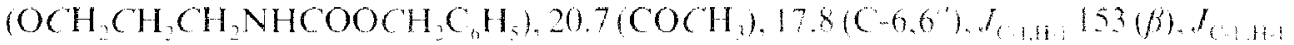

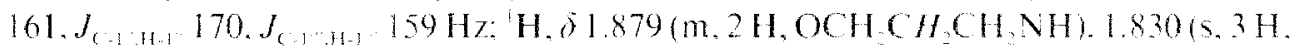
Ac), 1.361 and $1.277(2 \mathrm{~d}$, each $3 \mathrm{H}, 3 \mathrm{H}-6$ and $3 \mathrm{H}-6) . J_{0.6}, 0.2 \mathrm{~Hz}$.

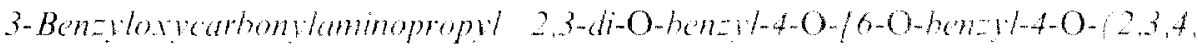

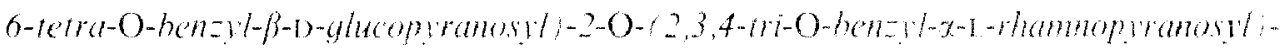

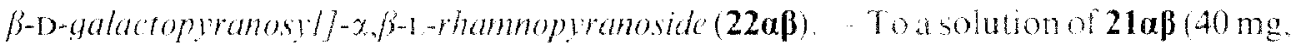
$23, \mu \mathrm{mol})$ in dichloromethane ( $2 \mathrm{~mL}$ ) and methanol $(3 \mathrm{~mL})$ was added sodium methoxide to $\mathrm{pH} 10$. After stirring for $48 \mathrm{~h}$. the mixture was neutralised wh Dowex- $50\left(\mathrm{H}^{\mathrm{H}}\right)$ resin. filtered, and concentrated. Column chromatography (95.5 dichoromethane ethy acetate) of the residue gave $220 \beta$, isolated as a syrup $(38 \mathrm{mg} .98 \%), \quad 0.48(92.8$ dichloromethane ethylacetate) ${ }^{3} \mathrm{C}-\mathrm{N}$ m.r. data $(\mathrm{CDCl}): 8156.3(\mathrm{NHCOOCH}, \mathrm{H})$ 139.3137 .0 and $128.5126 .7\left(\mathrm{C}_{6} \mathrm{H}_{4} \mathrm{CH}_{2} \mathrm{O}\right), 1(14.4(\mathrm{C}-1), 101.3(\mathrm{C}-1 / 3), 100.7(\mathrm{C}-1)$ $97.2\left(\mathrm{C}^{\prime \prime}\right) .38 .2$ and $29.6(\mathrm{OCH}, \mathrm{CH}, \mathrm{CH}, \mathrm{NH}), 18.0$ and $178(\mathrm{C}-6.6 \%)$

Anal Calc. for $\mathrm{C}_{14:} \mathrm{H}_{1:} \mathrm{NO}_{3}: \mathrm{C} .73 .02 ; \mathrm{H} .6 .71$. Found C. $72.58: \mathrm{H} .7 .14$

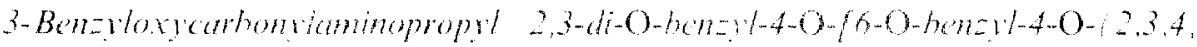

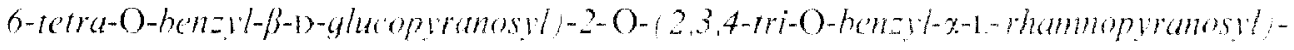

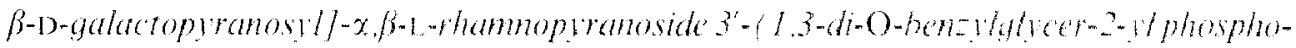
nate (23aß). - Compound $27(20 \mathrm{mg} .46 \mu \mathrm{mol})$ and $22 \alpha \beta(38 \mathrm{mg} .22$ famol) were co-concentrated in pyridine $(2 \times 5 \mathrm{~mL})$, and the residue was dissolved in dry pyridine $(4$ $\mathrm{mL}$ ). Pivaloyl chloride ( $9,4.73,4 \mathrm{~mol}$ ) was added the mixture was stired for 16 b at room temperature. water was added, and the mixture was diluted wiblichloromethane $(75 \mathrm{~mL})$, washed with $\mathrm{n}$ triethylammonium hydrogenarbonate $(2 \times 15 \mathrm{~mL})$. dried $\left(\mathrm{Na}_{2} \mathrm{SO}_{4}\right)$. filtered, and concentrated. Column chromatography $92: 8$ dichloromethane ethyl acetate) of the residue yielded $23 \alpha \beta$, isolated as a syrup $(37 \mathrm{mg} .82 \% 0), R_{0} 0.48(92.8$ dichloromethane ethyl acetate) ' H-N.m.r. data for $23 \beta(C D C l)=06.998\left(0.0 .5 \mathrm{H} . J_{n}\right.$ $720 \mathrm{~Hz} . \mathrm{P} \mathrm{H}$ ) and $6.796\left(\mathrm{~d}, 0.5 \mathrm{H}, J_{\mathrm{H}, \mathrm{P}}, 727 \mathrm{~Hz} . \mathrm{P} . \mathrm{H}\right)$ of two enantioners.

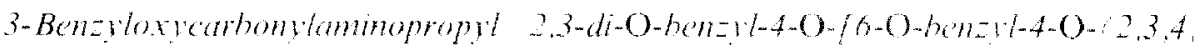

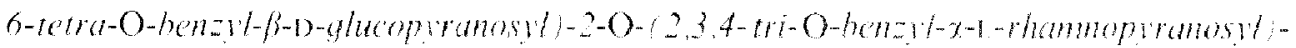


$\beta$-D-galactopyranosyl $/-\alpha, \beta$-L-rhamnopyranoside 3 - (1,3-di-O-benzylglycer-2-yl triethyl-

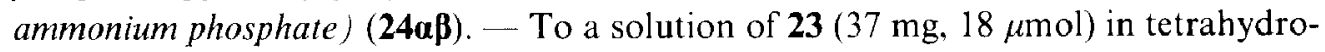
furan $(2 \mathrm{~mL})$ was added $0.35 \mathrm{M}$ iodine in $2: 1$ water-pyridine $(115 \mu \mathrm{L})$. After $3 \mathrm{~h}, \mathrm{t} .1 . \mathrm{c}$. (92:8 dichloromethane ethyl acetate) indicated the total disappearance of phosphonate 23, and a new product with $R_{\mathrm{F}} 0$ was observed. The excess of iodine was destroyed with aqueous $5 \%$ sodium hydrogensulfite $(10 \mathrm{~mL})$, and the mixture was diluted with dichloromethane $(50 \mathrm{~mL})$, washed with $\mathrm{M}$ triethylammonium hydrogencarbonate $(2 \times$ $15 \mathrm{~mL})$, dried $\left(\mathrm{Na}_{2} \mathrm{SO}_{4}\right)$, filtered, and concentrated. Column chromatography $(2: 1$

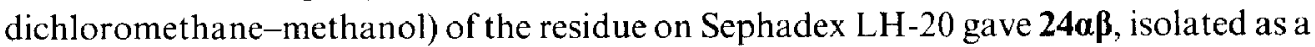
syrup $(35 \mathrm{mg}, 91 \%)$. N.m.r. data $\left(\mathrm{CDCl}_{3}\right):{ }^{13} \mathrm{C}, \delta 156.3\left(\mathrm{NHCOOCH} \mathrm{C}_{6} \mathrm{H}_{5}\right), 139.6$ 138.1 and $129.0-126.8\left(C_{6} \mathrm{H}_{5} \mathrm{CH}_{2} \mathrm{O}\right), 102.5\left(\mathrm{C}-1^{\prime \prime \prime}\right), 101.3(\mathrm{C}-1 \beta), 100.4\left(\mathrm{C}-1^{\prime}\right), 97.7$ $\left(\mathrm{C}-\mathrm{I}^{\prime \prime}\right), 44.8\left[\mathrm{NH}\left(\mathrm{CH}_{2} \mathrm{CH}_{3}\right)_{3}\right], 38.1$ and $29.6\left(\mathrm{OCH}_{2} \mathrm{CH}_{2} \mathrm{CH}_{2} \mathrm{NH}\right), 17.8\left(\mathrm{C}-6,6^{\prime \prime}\right), 8.1$ $\left[\mathrm{NH}\left(\mathrm{CH}_{2} \mathrm{CH}_{3}\right)_{3}\right] ;{ }^{31} \mathbf{P}, \delta-0.06$.

3-Aminopropyl 4-O-(4-O- $\beta$-D-glucopyranosyl-2-O- $\alpha-\mathrm{L}-$ rhamnopyranosyl- $\beta$-D-galactopyranosyl)- $\beta$-L-rhamnopyranoside 3 -(glycer-2-yl sodium phosphate) (25ß). -- To a solution of 24 ( $35 \mathrm{mg}, 16 \mu \mathrm{mol})$ in 1:2:2 ethyl acetate-2-propanol-methanol $(5 \mathrm{mI})$ was added $10 \% \mathrm{Pd}-\mathrm{C}(40 \mathrm{mg})$. Hydrogenolysis was performed at $4 \mathrm{~kg} / \mathrm{m}^{2}$ for $48 \mathrm{~h}$, the mixture was filtered and concentrated, and a solution of the residue in water was passed through a column of Dowex- $50\left(\mathrm{Na}^{+}\right)$resin, then lyophilised to yield 25 , isolated as a white powder ( $8 \mathrm{mg}, 57 \%$ ). The ${ }^{1} \mathrm{H}$-n.m.r. spect rum of 25 indicated mainly the $\beta$ anomer together with $\alpha$ anomer and degradation products (totalling $\sim 30 \%$ ). Final purification to yield $25 \beta$ was performed by HPAE-PAD chromatography using CarboPac PA 1 on a Dionex-LC system ${ }^{45}$ by elution with $0.1 \mathrm{M} \mathrm{NaOH}$ for $5 \mathrm{~min}$, followed by a linear gradient of $0 \rightarrow 600 \mathrm{~mm} \mathrm{NaOAc}$ in $0.1 \mathrm{M} \mathrm{NaOH}$ for $30 \mathrm{~min}$, and $600 \mathrm{~mm} \mathrm{NaOAc}$ for $5 \mathrm{~min}$. The major fraction, cluted at $9 \mathrm{~min}$, was collceted, neutralised with $0.1 \mathrm{M} \mathrm{HCl}$, and desalted. N.m.r. data $\left(\mathrm{D}_{2} \mathrm{O}\right):{ }^{13} \mathrm{C}, \delta 103.8,102.7,102.1$, and $101.1\left(\mathrm{C}-1,1^{\prime}, 1^{\prime \prime}, 1^{\prime \prime \prime}\right), 62.7(\mathrm{C}-1,3$ glycerol $), 62.1$ and $61.9\left(\mathrm{C}-6^{\prime}, 6^{\prime \prime \prime}\right), 68.6,39.0$, and $28.2\left(\mathrm{OCH}_{2} \mathrm{CH}_{2} \mathrm{CH}_{2} \mathrm{NH}_{2}\right), 18.5$ and $17.8\left(\mathrm{C}-6,6^{\prime \prime}\right) ;{ }^{31} \mathrm{P}, \delta 0.47 ;{ }^{1} \mathrm{H}$, see Table $\mathrm{I}$.

1,3-Di-O-Benzylglycerol 2-(triethylammonium phosphonate) (27). - To a solution of 1,3-di-O-benzylglycerol $(26 ; 0.96 \mathrm{~g}, 3.52 \mathrm{mmol})$ in acetonitrile $(40 \mathrm{~mL})$ was added pyridine $(12.5 \mathrm{~mL})$ and 2-chloro-4H-1,3,2-benzodioxaphosphorin-4-one $(1.0 \mathrm{~g}, 4.9$ mmol). After $1 \mathrm{~h}, \mathrm{t} . \mathrm{l} . \mathrm{c}$. (9:1 dichloromethane-ethyl acetate) showed the disappearance of $26\left(R_{\mathrm{F}} 0.9\right)$ and a new spot at $R_{\mathrm{r}} 0$. Water $(3 \mathrm{~mL})$ was added, and the solution was diluted with $99: 1$ dichloromethane-triethylamine $(200 \mathrm{~mL})$, washed with $\mathrm{m}$ triethylammonium hydrogencarbonate $(2 \times 30 \mathrm{~mL})$, dried $\left(\mathrm{Na}_{2} \mathrm{SO}_{4}\right)$, filtered, and concentrated. Column chromatography (80:20:0.1 dichloromethane acetone-triethylamine, followed by 92:8:0.1 dichloromethane-methanol-triethylamine) of the residue yielded 27, isolated as a syrup $\left(1.38\right.$ g, 89\%). N.m.r. data $\left(\mathrm{CDCl}_{3}\right):{ }^{13} \mathrm{C}, \delta 138.0$ and $127.9-127.1$ $\left(\mathrm{C}_{6} \mathrm{H}_{5} \mathrm{CH}_{2} \mathrm{O}\right), 72.8\left(\mathrm{PhCH}_{2} \mathrm{O}\right), 71.8(\mathrm{~d}, \mathrm{C}-2), 70.3(\mathrm{~d}, \mathrm{C}-1,3), 45.0\left[\mathrm{~N}\left(\mathrm{CH}_{2} \mathrm{CH}_{3}\right)_{3}\right], 8.0$ $\left[\mathrm{N}\left(\mathrm{CH}_{2} \mathrm{CH}_{3}\right)_{2}\right],{ }^{2} J_{\mathrm{C}-2 . \mathrm{P}} 5.3,{ }^{3} J_{\mathrm{C}-1 . \mathrm{P}}={ }^{3} J_{\mathrm{C}-3 . \mathrm{P}}=3.8 \mathrm{~Hz} ;{ }^{1} \mathrm{H}, \delta 7.293-7.222(\mathrm{~m}, 10 \mathrm{H}, 2 \mathrm{Ph})$, $6.977(\mathrm{~d}, 1 \mathrm{H}, \mathrm{P}-\mathrm{H}), 4.595(\mathrm{~m}, 1 \mathrm{H}, \mathrm{H}-2), 4.539$ and 4.503 ( $2 \mathrm{~d}$, each $\left.2 \mathrm{H}, 2 \mathrm{PhC} \mathrm{H}_{2} \mathrm{O}\right)$, $3.660(\mathrm{~m}, 4 \mathrm{H}, \mathrm{H}-1,1,3,3), 2.959\left[\mathrm{q}, 6 \mathrm{H}, \mathrm{N}\left(\mathrm{CH}_{2} \mathrm{CH}_{3}\right)_{3}\right], 1.219\left[\mathrm{t}, 9 \mathrm{H}, \mathrm{N}\left(\mathrm{CH}_{2} \mathrm{CH}_{3}\right)_{2}\right],{ }^{1} J_{\mathrm{H}, \mathrm{P}}$ $635, J_{1,2}=J_{2,3}=5.3,{ }^{3} J_{\mathrm{H}-2, \mathrm{P}} 10.6, J_{\mathrm{CH}_{2} \mathrm{CH}_{3}} 7.3 \mathrm{~Hz} ;{ }^{31} \mathrm{P}, \delta 4.46(\mathrm{dd}),{ }^{1} J_{\mathrm{H} . \mathrm{P}} 635,{ }^{3} J_{\mathrm{H}-2, \mathrm{P}} 10.6 \mathrm{~Hz}$. 
The capsular polysaccharide of Streptococcus phemonice $23 \mathrm{~F}$ was a gift of 1 ) $\mathrm{H}$. Snippe (Department of Experimental Immunology, Utrecht University). We thank Dr. $H$. Voshol for the purification of the polysaccharide and the spacer-contaning letrasaccharide 25B, and Drs. P. de Waard and B. Leellang for recording the $500-\mathrm{MH} / 2 \mathrm{D} H$ and RESED n.m.r. spectra

\section{RFFFRENCFS}

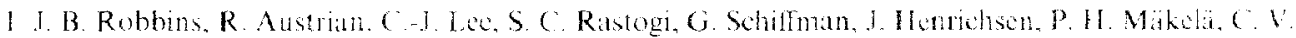

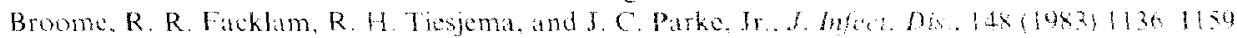

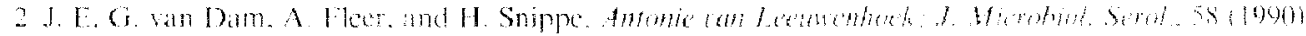
147

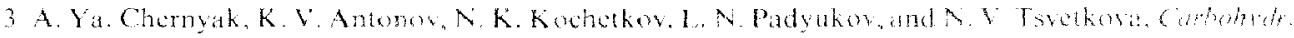
Res. $141(1985) 190212$

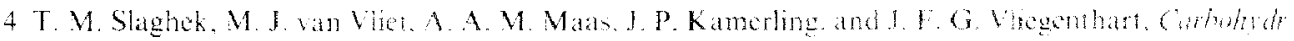
Ro. $195(1989) 7586$

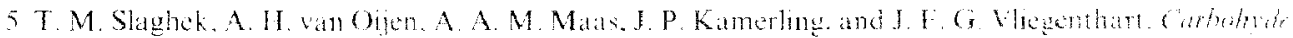
$R(x .2070090) 237248$.

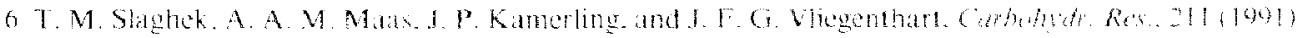
$25 \quad 39$.

7 H. Paulsen and B. Helpap. Camolnde, Re, $186(1989) 183205$

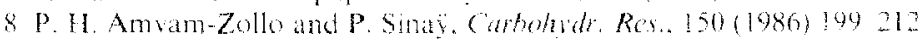

9 N. K. Kochetkov. N. F. Nifantet, and L. V. Backinowsky, Termbedrom, 43 (1987) 3109 3121

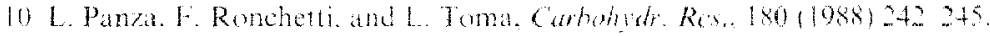

11 T. Suganata and T. Igarahi. Carbohdr. Rex.1721988) 196207

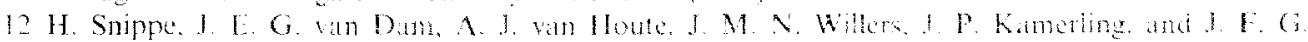
Vhegenthart. Infert. Immmi. 4311963$) 842 \times 44$

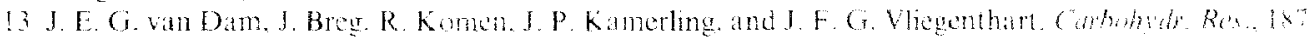
(1989) $267 \cdots 286$.

14 J. F. G. van Dam. Thesis. Utrecht Lniversity. The Netherlands, 1988

15 A. Roy and V. Roy. Camblir. Ros.. $126(1984) 271277$

16 C. Jones, Curbohdr. Rex. 1391985)7583.

17 I. C. Richards and M. B. Perry, Bioghm. Coll Biol. 66 (1988) 758771 .

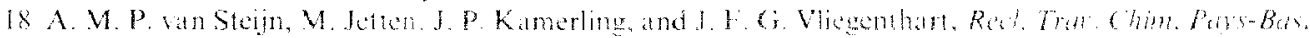
$108(1989) 374.383$

19 A. K. Ray. L. B. Maddali. A Roy and N. Roy. Carbohydr. Re's. 197 (1090)93 lon

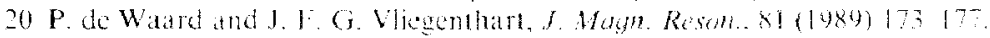

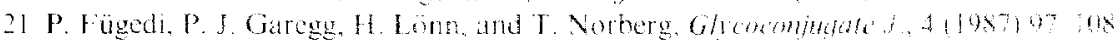

22 H. Lön. Thesis, Unversity of Stockhom, Sxaden. 1984

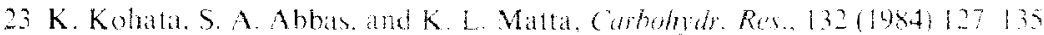

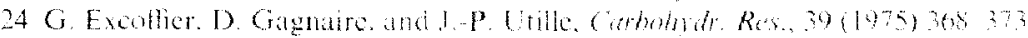

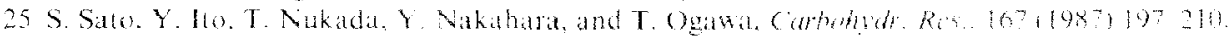

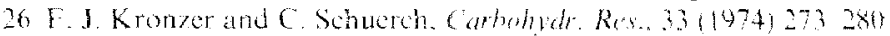

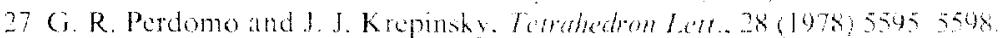

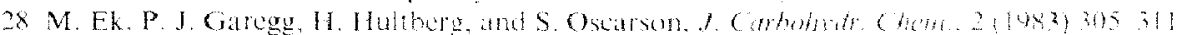

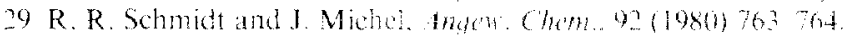

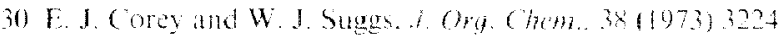

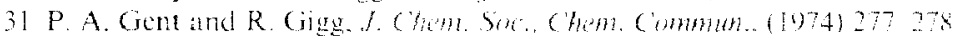

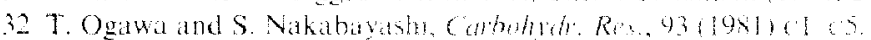

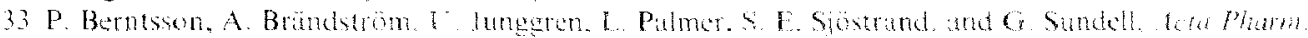
Suter, $14(1977) 229236$

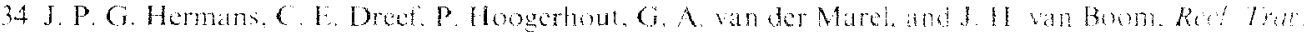

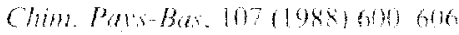


35 H. Paulsen, Angew. Chem. Int. Ed. Engl, 21 (1982) 155-173.

36 S. Sato, M. Mori, Y. Ito, and T. Ogawa, Carbohydr. Res., 155 (1986) C6-c10.

37 H. Paulsen and W. Kutscher, Carbohydr. Res., 120 (1983) 2542.

38 F. Weygand and H. Ziemann, Justus Liebigs Ann. Chem., 657 (1962) 179-198.

39 K. Bock, I. Lundt, and C. Pedersen, Tetrahedron Lett., (1973) 1037-1040.

40 E. de Vroom, C. E. Dreef, H. van den Elst, G. A. van der Marel, and J. H. van Boom, Recl. Trav. Chim. Pays-Bas, 107 (1988) 592-595.

41 P. Westerduin, G. H. Veeneman, G. A. van der Marel, and J. H. van Boom, Tetrahedron Lett., 27 (1986) 6271-6274.

42 J.P. G. Hermans, E. de Vroom, C. J. J. Elie, G. A. van der Marel, and J. H. van Boom, Recl. Trav. Chim. Pays-Bas, 105 (1986) $510-511$.

43 M. Lindberg and T. Norberg, J. Carbohydr. Chem., 7 (1988) $749 \ldots 755$.

44 L. J. J. Hronowski, W. A. Szarek, G. W. Hay, and W. T. Depew, Carbohydr. Res., 190 (1989) $203-218$.

45 R. R. Townsend, M. R. Hardy, O. Hindsgaul, and Y. C. Lee, Anal. Biochem., 174 (1988) 459-470.

46 D. Marion and K. Wüthrich, Biochem. Biophys. Res. Commun., 113 (1983) 967-974.

47 A. Bax and D. G. Davis, J. Magn. Reson.,65 (1985) 355-360.

48 C. Laffite, A. M. Nguyen Phuoc Du, F. Winternitz, R. Wylde, and F. Pratviel-Sosa, Carbohydr. Res., 67 (1978) 91-103. 